\title{
Caenorhabditis elegans ETR-1/CELF has broad effects on the muscle cell transcriptome, including genes that regulate translation and neuroblast migration
}

\author{
Matthew E. Ochs ${ }^{1}$, Rebecca M. McWhirter ${ }^{2}$, Robert L. Unckless' ${ }^{1}$, David M. Miller $\|^{2}{ }^{2}$ and Erik A. Lundquist ${ }^{*}$
}

\begin{abstract}
Migration of neuroblasts and neurons from their birthplace is central to the formation of neural circuits and networks. ETR-1 is the Caenorhabditis elegans homolog of the CELF1 (CUGBP, ELAV-like family 1) RNA-processing factor involved in neuromuscular disorders. etr-1 regulates body wall muscle differentiation. Our previous work showed that etr-1 in muscle has a non-autonomous role in neuronal migration, suggesting that ETR-1 is involved in the production of a signal emanating from body wall muscle that controls neuroblast migration and that interacts with Wnt signaling. etr-1 is extensively alternatively-spliced, and we identified the viable etr-1 (lq61) mutant, caused by a stop codon in alternatively-spliced exon 8 and only affecting etr-1 isoforms containing exon 8. We took advantage of viable etr1(Iq61) to identify potential RNA targets of ETR-1 in body wall muscle using a combination of fluorescence activated cell sorting (FACS) of body wall muscles from wild-type and etr-1(Iq61) and subsequent RNA-seq. This analysis revealed genes whose splicing and transcript levels were controlled by ETR-1 exon 8 isoforms, and represented a broad spectrum of genes involved in muscle differentiation, myofilament lattice structure, and physiology. Genes with transcripts underrepresented in etr-1 (la61) included those involved in ribosome function and translation, similar to potential CELF1 targets identified in chick cardiomyocytes. This suggests that at least some targets of ETR-1 might be conserved in vertebrates, and that ETR-1 might generally stimulate translation in muscles. As proof-of-principle, a functional analysis of a subset of ETR-1 targets revealed genes involved in AQR and PQR neuronal migration. One such gene, lev11/tropomyosin, requires ETR-1 for alternative splicing, and another, unc-52/perlecan, requires ETR-1 for the production of long isoforms containing $3^{\prime}$ exons. In sum, these studies identified gene targets of ETR-1/CELF1 in muscles, which included genes involved in muscle development and physiology, and genes with novel roles in neuronal migration.
\end{abstract}

\section{Introduction}

Migration of neuroblasts and neurons is a key developmental process in the formation of neural circuits and networks. The CELF (CUGBP, ELAV-like family) class of RNA-binding proteins is implicated in a wide variety of

\footnotetext{
*Correspondence: erikl@ku.edu

1 Program in Molecular, Cellular, and Developmental Biology, Department of Molecular Biosciences, University of Kansas, Lawrence, KS 66045, USA Full list of author information is available at the end of the article
}

neuromuscular and neurodegenerative disorders, including Myotonic Dystrophy type I (DMI) [1-7], the cardiac syndrome arrythmogenic right ventricular dysplasia [8], Alzheimer's disease [9], spinocerebellar ataxia type 8, and possibly fragile $\mathrm{X}$ syndrome [10, 11]. CELF proteins control mRNA processing, including alternative splicing [12-14], and regulation of translation [15], and mRNA transcript stability [16, 17]. CELF protein structure is characterized by three RNA-recognition motifs (RRM) with a non-conserved region between RRM2 and RRM3,

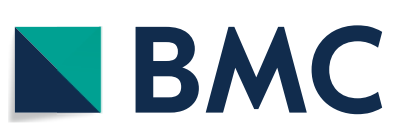

(c) The Author(s) 2021. Open Access This article is licensed under a Creative Commons Attribution 4.0 International License, which permits use, sharing, adaptation, distribution and reproduction in any medium or format, as long as you give appropriate credit to the original author(s) and the source, provide a link to the Creative Commons licence, and indicate if changes were made. The images or other third party material in this article are included in the article's Creative Commons licence, unless indicated otherwise in a credit line to the material. If material is not included in the article's Creative Commons licence and your intended use is not permitted by statutory regulation or exceeds the permitted use, you will need to obtain permission directly from the copyright holder. To view a copy of this licence, visit http://creativecommons.org/licenses/by/4.0/. The Creative Commons Public Domain Dedication waiver (http://creativeco mmons.org/publicdomain/zero/1.0/) applies to the data made available in this article, unless otherwise stated in a credit line to the data. 
an RRM organization conserved across CELF protein family members [16].

Vertebrate genomes encode up to six CELF molecules [18], CELF1-6. In C. elegans, there are two CELF genes, etr-1 (most similar to CELF1-2) [19, 20], and unc-75 (most similar to CELF3-6) [21]. unc-75 controls alternative splicing events predominantly in the nervous system [21-25]. ETR-1 is involved in muscle development, as knockdown of ETR-1 results in severe muscle disorganization and embryonic lethality [19]. ETR-1 also controls cell corpse engulfment in the germline [26], and influences neuronal migration non-autonomously from body wall muscle [20].

ETR-1 acts in muscle to guide the long-range migration of the Q neuroblast descendants in C. elegans [20]. The Q neuroblasts, QR and QL, are bilaterally symmetrical cells that undergo similar divisions and stereotypical migrations (reviewed in [27]). QR is born on the right side of the animal and QR descendants migrate anteriorly. QL is born on the left side of the animal and QL descendants migrate posteriorly. Both QR and QL produce three functional neurons, and two apoptotic bodies. QR produces $A Q R, A V M$ and SDQR, with AQR migrating the furthest, residing just posterior to the posterior pharyngeal bulb in the anterior deirid ganglion. QL produces PQR, PVM and SDQL, with $P Q R$ migrating the furthest, residing posterior to the anus in the phasmid ganglion [28-30]. Due to the stereotypical migrations, the $\mathrm{Q}$ neuroblasts are a powerful system to identify migration defects and study the genetic mechanisms controlling migration. Initial QL and QR migrations are controlled by interactions between three receptor molecules UNC-40/DCC, PTP-3/ LAR and MIG-21, and the Fat-like Cadherins CDH-3 and CDH-4 [31-35]. Migration of the Q descendants is controlled by Wnt signaling along the anterior-posterior body axis [36-38]. Previous studies have implicated body wall muscle cells as sources of migration factors for the $\mathrm{Q}$ neuroblast descendants [39, 40].

The etr-1(lq61) mutation was isolated in a forward genetic screen for $\mathrm{AQR}$ and $\mathrm{PQR}$ migration defects [20]. lq61 introduces a premature stop codon in alternativelyspliced exon 8, which is present in a subset of etr-1 isoforms. Total knockdown of etr-1 resulted in embryonic lethality with muscle defects [19], yet etr-1(lq61) animals are viable and fertile, consistent with lq61 being a hypomorphic mutation. Etr-1 is expressed in all cells of the embryo [41], but acts in the muscle cells in a non-autonomous manner to control AQR and PQR migrations [20]. Furthermore, etr-1(lq61) interacts genetically with Wnt mutations in $\mathrm{AQR}$ and $\mathrm{PQR}$ migration.

As etr-1(lq61) is a viable and fertile mutation, it presented a unique opportunity to identify gene targets of a CELF1/2 family member in muscles, and to define target genes that contribute to the non-autonomous control of $A Q R$ and PQR migration by ETR-1. We used fluorescence activated cell sorting of $C$. elegans body wall muscle cells from wild-type and etr-1(lq61) mutants, combined with RNA-seq, to define muscle-expressed genes with alternative exon usage and transcript accumulation and in etr-1(lq61) mutants. This analysis revealed genes involved in myofilament lattice assembly and adhesion, and muscle physiology. Genes with underrepresented transcripts in etr-1(lq61) were involved in translation and ribosome function. As proof of principle, a pilot functional screen identified new genes for AQR and $\mathrm{PQR}$ migration, including unc-52/perlecan and lev11/tropomyosin. ETR-1 targets, including lev-11/tropomyosin and genes involved in translational and ribosome function, were also identified in vertebrates [16, 17], suggesting a deep evolutionary conservation of CELF targets and potentially conserved molecular mechanisms of CELF1/2 function from C. elegans to vertebrates.

\section{Results}

\section{Fluorescent-activated cell sorting of muscle cells and RNA} seq

myo-3::gfp-expressing body wall muscle cells from synchronized early L1 larvae were isolated by FACS as described in Material and Methods and in [42, 43] (Fig. 1). Muscles were isolated from the wild-type (N2) strain and etr-1(lq61) mutants. Three biological replicates for each genotype were isolated. RNA was also isolated from triplicate samples of non-dissociated L1 larvae for the all-cell control group.

A total of 12 RNA-seq libraries were constructed, representing three biological replicates of both $\mathrm{N} 2$ and etr1(lq61) with both sorted muscle cells and whole L1 larval stage cells (see Materials and Methods). Paired-end 150bp reads were generated from each of the 12 samples using the Illumina Nextseq550 platform. FASTQ files can be accessed in the Sequence Read Archive, Project number PRJNA733501.

\section{A muscle cell transcriptome defined by RNA-seq}

We used DEseq2 to identify genes with significant differential expression in wild-type muscle cells compared to all L1 stage wild-type cells (Supplemental File 1). There were 3718 protein-coding genes with significantly higher expression in muscle cells compared to all cells (log2fold change $\geq 0.5849$ (1.5x); $q \leq 0.05$ ), including many canonical muscle structure and function genes previously shown to be expressed in muscle (e.g. unc-15/paramyosin, unc-54/myosin, unc-95/paxillin, and the myofilament structure pat genes [44]. DEseq2 also identified etr-1 as being more highly expressed in muscle (Supplemental File $1 ; \log _{2}$ fold change $2.21 ; q=9.93 e^{-37}$ ). Differential 
A

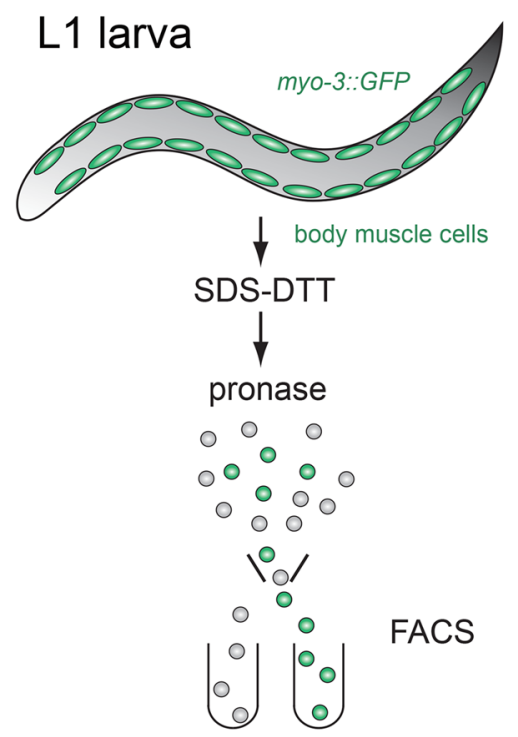

B

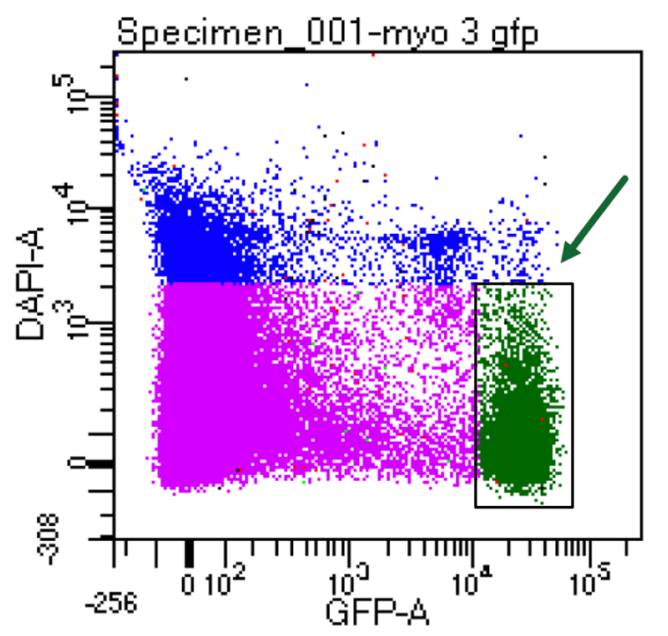

Fig. 1 FACS isolation of body muscle cells from wild type and etr-1 (lq61) mutant L1 larvae. A Synchronized L1-stage larvae were dissociated by successive treatments with SDS-DTT and pronase to release myo-3::gfp labeled body muscle cells for isolation by FACS. B FACS scatter plot. Viable and brightly labeled myo-3::GFP marked cells (arrow) were captured for RNA extraction. Damaged cells were excluded by DAPI staining

exon usage using DEXseq showed that multiple etr-1 exons showed significantly increased expression in muscle compared to all cells as a whole (Fig. 2 A). The etr-1 locus is extensively alternatively spliced [20, 26], and isoforms with exon 8 are required in muscles for $Q$ neuroblast descendant migration [20]. Exon 8 expression was increased in muscles compared to all cells as a whole (Fig. 2A), suggesting that isoforms with exon 8 might be more abundant in muscles compared to all cells as a whole. Expression of 8763 protein coding genes was significantly reduced in muscle cells compared to all cells (Supplemental File 1). Differential expression of non-coding RNAs and pseudogenes is presented in Supplemental File 1.

\section{Genes with exon usage affected by etr-1(Iq61) encode molecules involved in myofilament lattice structure and attachment, and muscle physiology}

CELF family proteins are known to regulate splicing $[12,45]$, and we endeavored to determine the effects of ETR-1/CELF on the muscle transcriptome, including splicing. We compared exon representation across the genome in wild-type and etr-1(lq61) mutant muscle cells using the Bioconductor package DEXseq [46] (see Materials and Methods). Across the genome, there were 242 protein-coding genes and seven non-coding RNA genes with at least one exon significantly differentially represented in etr-1(lq61) muscle compared to wild-type $(q \leq 0.05)$ (Supplemental File 2).

The etr-1(lq61) mutation is a premature stop codon in alternatively-spliced exon 8 [20]. Exon 8 was significantly underrepresented in etr-1(lq61) muscle compared to wild-type (Fig. 2B), suggesting that transcripts containing exon 8 are reduced in $e t r-1(l q 61)$ muscle cells, as predicted. Exon 13 was also significantly underrepresented in etr-1(lq61). Possibly, transcripts with exon 8 might preferentially contain exon 13 . Alternatively, ETR-1 containing exon 8 might be involved in the regulation of processing of etr-1 exon 13 .

We used the Database for Annotation, Visualization and Integrated Discovery (DAVID) $[47,48]$ to perform a gene ontology term (GO term) analysis on this gene set that showed differential exon representation in etr-1(lq61) mutant muscle compared to wild-type muscle, including both over-and underrepresented exons in wild-type compared to etr-1(lq61) (see Materials and Methods). We analyzed GO terms for the three categories: biological process (BP), cellular component (CC) and molecular function (MF) (Supplemental File 3). The six most significantly enriched GO terms in each category are shown in Fig. 3. These include GO terms associated with myofilament lattice formation and function (e.g. striated muscle myosin thick filament assembly, locomotion, $M$ band, striated muscle thin filament assembly, striated muscle dense 

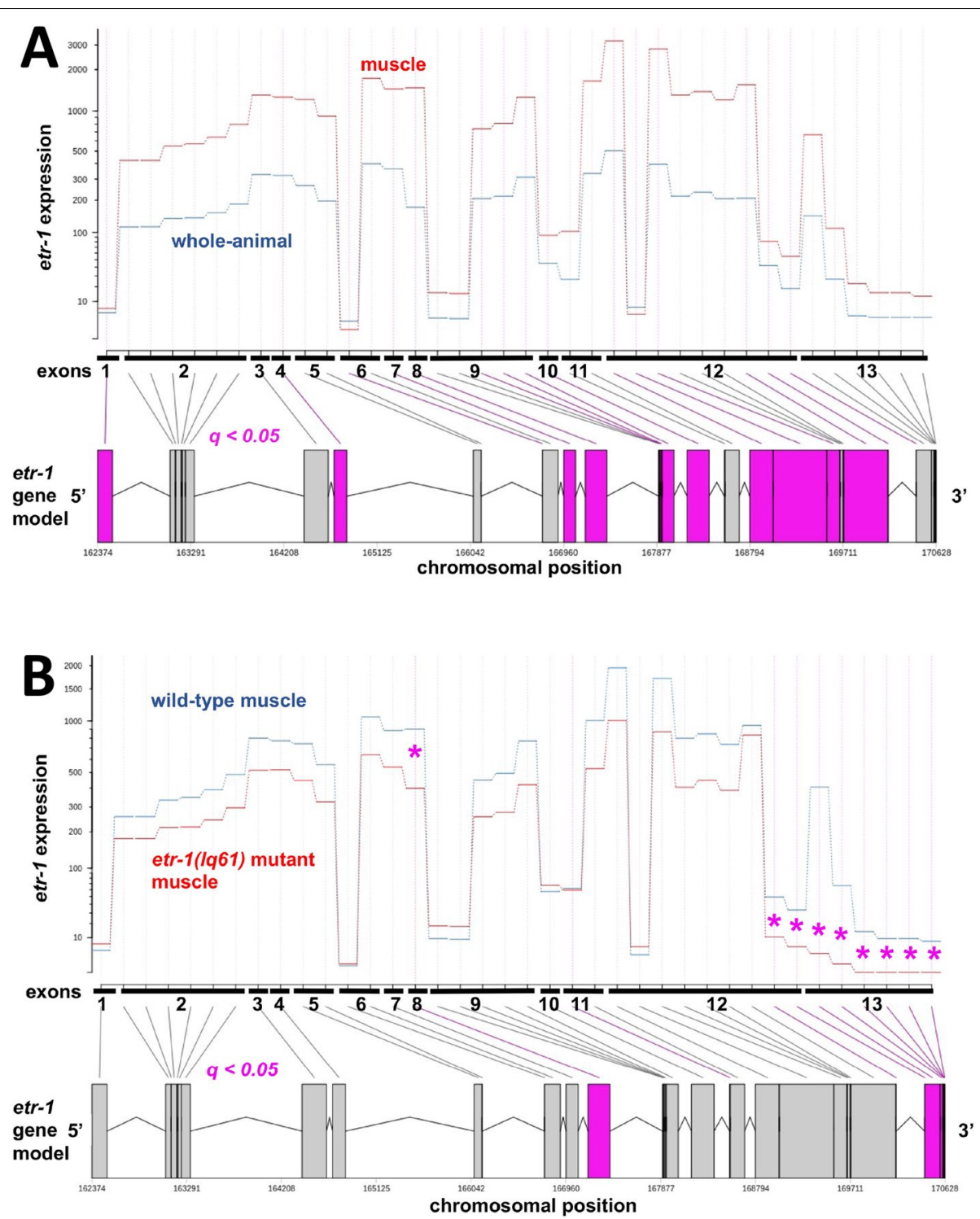

Fig. 2 Output of DEXSeq showing differential exon usage. A Exon usage of etr-1 comparing wild-type muscle to wild-type whole animal. The red line represents muscle expression, the blue line represents whole animal expression. Exons that are significantly different $(q \leq 0.05)$ are in purple and indicated with purple asterisks. Etr-1 exons were overrepresented in muscle cells, including exon 8 harboring the lq61 mutation. B Exon usage of etr-1 comparing etr-1(lq61) muscle cells to wild-type muscle cells. The red line represents etr-1(lq61) expression and the blue line represents wild-type expression. Etr-1 exons generally were underrepresented in etr-1(lq61), with exon 8 and the $3^{\prime}$ exon significantly so (purple). Some exons have multiple comparisons because of different $5^{\prime}$ and $3^{\prime}$ exon boundaries for the exon in transcript isoforms annotated in the .gtf file. For example, exon 2 has six distinct $5^{\prime}$ and $3^{\prime}$ boundaries in different annotated isoforms, whereas exons 3 and 4 have a single $5^{\prime}$ and $3^{\prime}$ boundary in all annotated isoforms

body, I band, actin filament, and actin filament binding). Also included are muscle physiology GO terms (e.g. voltage gated ion channel activity, calcium ion binding, voltage gated potassium channel activity, and kinase activity). These are consistent with the previously-reported effects of etr-1 RNAi knockdown on muscle development and attachment [19]. GO terms of apoptotic process and reproduction are also enriched, consistent with a known role of etr-1 in germline development and engulfment of germ cell apoptotic corpses [26].

Genes with transcript accumulation affected by etr-1(lq61) encode molecules involved in translation and ribosome function

The CELF-family proteins control transcript stability $[12,13,45]$. We used stringTie and DEseq2 to identify 


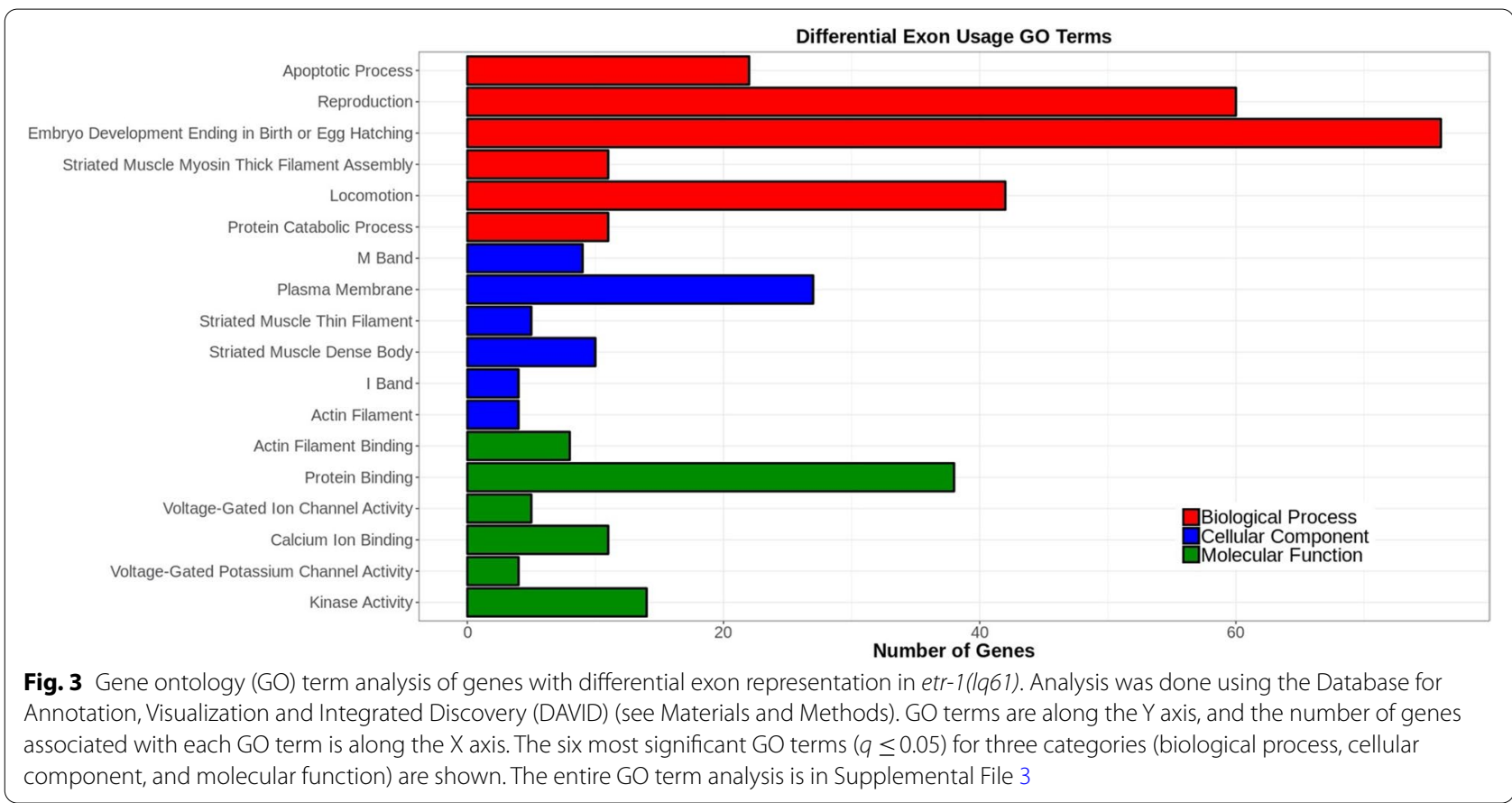

transcripts with differential accumulation in wild-type versus etr-1(lq61) muscle cells (see Materials and Methods). We identified transcripts that were differentially represented with a $\log _{2}$ fold change $\geq 1(2 \mathrm{x})$ and a false discovery rate less than $0.05(q \leq 0.05)$, to increase stringency given the large number of genes returned in this analysis (Supplemental File 4).

We identified 1180 transcripts representing 971 loci with altered accumulation in etr-1(lq61) (Fig. 4A), including coding and non-coding RNAs (Supplemental File 4). 506 loci had transcripts that were overrepresented, and 414 loci had transcripts that were underrepresented in etr-1(lq61) muscle cells (Fig. 4B). There were 51 loci in which some transcripts were overrepresented and some underrepresented (Fig. 4B and Table 1).

Gene Ontology enrichment analysis was conducted on four separate groups of these muscle genes with transcripts affected by etr-1(lq61) (Supplemental File 5): all genes; genes with transcripts that were only underrepresented; genes transcripts that were only overrepresented; and genes that had transcripts both over- and underrepresented. The six most significant GO terms for each group are shown in Fig. 5. Considering all genes, GO terms associated with translation and ribosomal function were apparent, as well as myofilament structure, muscle physiology, reproduction, and embryonic and larval growth (Fig. 5A). Genes with transcripts underrepresented in etr-1(lq61) were described by GO terms representing translation and ribosomal function (10 of the 18 top GO terms) (Fig. 5B). Genes with overrepresented transcripts were described by GO terms representing a broad cross section of cellular function, but translation and the ribosome were not among these (Fig. 5C). Genes with both over- and underrepresented transcripts were described by GO terms representing myofilament lattice and muscle physiology, and other cellular functions (Fig. 5D). In sum, this GO term analysis suggests that etr-1(lq61) influences a broad spectrum of cellular events in muscle, including myofilament lattice and muscle physiology, as well as translation and ribosomal function. Notably, genes involved in translation and ribosomal function are strongly represented among those with transcripts reduced in etr-1(lq61). A similar reduction of expression of genes involved in translation and ribosomal function was described after siRNA knock-down of CELF1 in chicken cardiomyocytes [17].

\section{Genes identified by both differential exon usage and transcript accumulation}

etr-1(lq61) affected transcript expression of 971 genes, and alternative exon representation of 244 genes. There were 102 genes shared between the 244 alternativelyspliced genes and the 971 genes that had differentiallyexpressed transcripts (Table 3), a significant association $(p \leq 0.0001)$. These might represent genes with transcripts for which ETR-1 controls both splicing and transcript accumulation. Alternately, the differential use of exons could influence the transcript accumulation DEseq2 algorithm, leading to under- or overrepresentation of transcripts by alternate exon usage. In any event, 


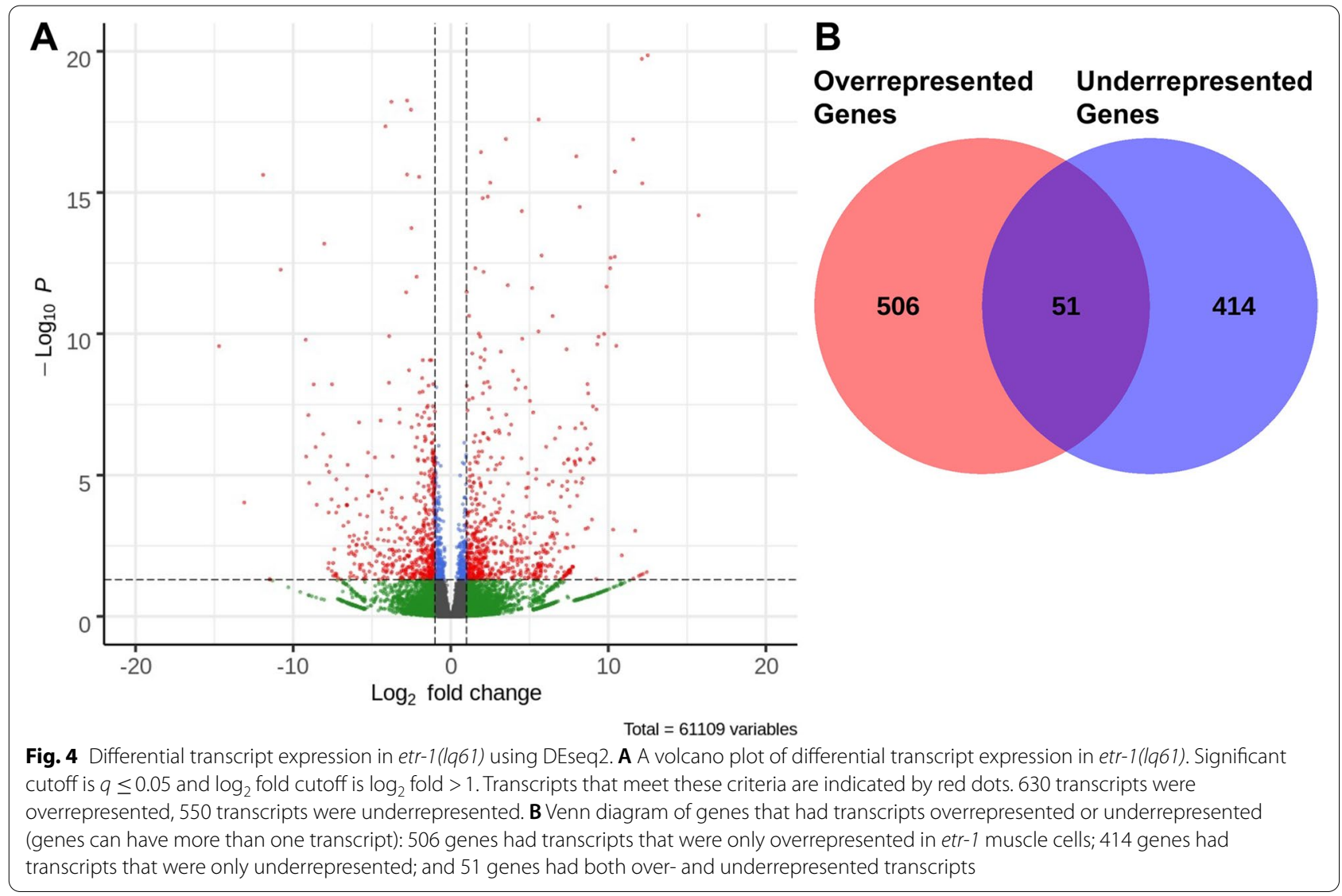

identification in both analyses suggests that ETR-1 might have strong effects on the transcripts of these genes.

\section{Genes affecting $A Q R$ and $P Q R$ migration}

This analysis suggests that ETR-1 regulates multiple aspects of muscle cell function, most notably myofilament lattice, muscle physiology, and translation and ribosomal function. Previous studies indicated that etr1(lq61) had a muscle-derived, non-autonomous effect on migration of $A Q R$ and $P Q R$ neurons [20]. Thus, ETR-1 might regulate a secreted signal from the muscles that directs $A Q R$ and $P Q R$ migration. Genetic analysis suggests this signal could act with or in parallel to Wnt signaling [20], which directs $A Q R$ and $P Q R$ migration [38].

We used feeding RNAi (see Materials and Methods) and mutants to knock down a subset of genes with known roles in cell migration, and genes that had transcripts both overrepresented and underrepresented in etr-1(lq61) muscles versus wild-type muscles (Table 1). $A Q R$ and $P Q R$ position in these animals was scored (see Materials and Methods) (Table 3). In unc-52, unc-71, and $s k n-1$, between 2 and $5 \%$ of $\mathrm{AQR}$ and PQR neurons migrated in the wrong direction, as evidenced by $A Q R$ in position 5 , and PQR in positions 1,2 , and 3 . UNC-52 is the basement membrane heparan sulfate proteoglycan Perlecan [49] and will be discussed in more detail below. UNC-71 is an ADAM metalloprotease that has been shown to act in anterior $\mathrm{Q}$ descendant migration [50, 51]. SKN-1 is an ortholog of the human NFE2L1 transcriptional regulator that controls a wide variety of developmental events including muscle differentiation [52, 53]. Some genes displayed defects in the ability of AQR and PQR to migrate (>10\%), but not directional defects, as evidenced by $A Q R$ in positions 2,3 , and 4 and $P Q R$ in position 4. Many genes displayed few $(<10 \%)$ or no defects in the ability of AQR and PQR to migrate. Thus, some genes with transcripts that are regulated by ETR-1 in muscle had instructional roles in directing $A Q R$ and $\mathrm{PQR}$ migration, and some had permissive roles in the ability of $A Q R$ and $P Q R$ to migrate.

\section{The heparan sulfate proteoglycan UNC-52 affects AQR and $\mathrm{PQR}$ migration}

unc-52 encodes the basement membrane heparan sulfate proteoglycan Perlecan and is involved in myofilament lattice attachment to the basement membrane [49]. unc-52 is extensively alternatively spliced [54, 55], including in the epidermis by CCAR-1 $[56]$ and MEC- $8[57,58]$ with 
Table 1 Genes with transcripts both overrepresented and underrepresented in etr-1 (lq61)muscle

unc-52

lev-11

daf-3

rpt-4

unc-22

dur-1

alp-1

fhod-1

$n t l-2$

ttn-1

yif-1

atn-1

egl-2

ceh-79

nsy-7

avr-14

mel-11

rme-1

nhre-61 pnk-1

sir-2.1

T10C6. 6

slo-2

spv-1

unc-73

lec-3

cup-5

T13H5. 1

unc-16

tnt-3

slo-1

F49E2.5

unc-36

ret-1

$d n j-5$

pqn-80

tax-6

F25E5.8 tag-151

mrp-1

mbk-2

T23E7. 2

ZK185.1

unc-68

\section{pdi-2}

pbrm-1

deb-1

add-2

\section{T07D3.9 tep-1}

consequences on hemidesmosome formation, muscle attachment and mechanosensory neuron function.

We found that etr-1(lq61) affected unc-52 transcript expression in muscle cells. unc-52 was identified in both exon representation by DEXseq and transcript accumulation by DEseq2 (Table 2). In etr-1(lq61) muscle cells, some $5^{\prime}$ exons were significantly overrepresented and $3^{\prime}$ exons significantly underrepresented compared to wild-type muscle cells. The far $5^{\prime}$ exons (see Fig. 6A) predicted for $u n c-52$ were not highly expressed in muscle in either background. We visualized unc-52 splice junctions using the Sashimi plot function in the Integrated Genome Viewer (see Materials and Methods) (Fig. 6B). In wildtype animals, $3^{\prime}$ exons were well-represented (Fig. 6B). In etr-1(lq61), $3^{\prime}$ exons were significantly underrepresented and $5^{\prime}$ exons significantly overrepresented. These 
data suggest that ETR-1 proteins that include exon 8 are required to produce the long isoforms of $u n c-52$ containing the $3^{\prime}$ exons in muscles.

As described above, unc-52(RNAi) resulted in AQR and PQR directional migration defects (Table 1). For RNAi, we utilized the ZC101.2 Source Bioscience II-9A20 clone, which is located located in the $3^{\prime}$ region of $u n c-52$, the region that is underrepresented in etr-1(lq61) muscles (Fig. 6B). unc-52(RNAi) animals displayed the paralyzed, arrested at two-fold stage (Pat) phenotype similar to strong loss-of-function alleles of unc-52 (Fig. $7 \mathrm{~A}$ and B) [59]. Notably, etr-1(lq61) animals, which do not express the $3^{\prime}$ exons from this $u n c-52$ region in muscle, do not show the Pat phenotype. The viability of etr-1(lq61) mutants could be.

due to a muscle-specific effect of ETR-1 on unc-52, with unc-52 long isoforms with $3^{\prime}$ exons expressed and functional in other tissues (e.g. hypodermis) in etr1(lq61) mutants. Alternatively, it is possible that the phenomenon of RNAi amplification [60] results in all unc-52 isoforms being affected by RNAi, in which case conclusions about the roles of the $3^{\prime}$ exons cannot be drawn.

Despite embryonic lethality and the Pat phenotype, $A Q R$ and $P Q R$ were visible in unc-52(RNAi) arrested Pat animals. $A Q R$ and $P Q R$ displayed defects in the ability of $\mathrm{AQR}$ and $\mathrm{PQR}$ to migrate (Fig. $7 \mathrm{~A}$ and $\mathrm{B}$ ) as well as defects in direction of migration (Table 3). In sum, ETR-1 proteins that contain exon 8 are required for the accumulation of long isoform transcripts of $u n c-52$ containing $3^{\prime}$ exons in muscles. Targeting $u n c-52$ with RNAi resulted in $A Q R$ and PQR defects, suggesting a role in $A Q R$ and PQR migration.

\section{RNAi knockdown of lev-11 results in $\mathrm{AQR}$ and $\mathrm{PQR}$ migration defects}

lev-11 was also identified by both exon representation and transcript accumulation in etr-1(lq61) (Table 2). lev11 encodes a tropomyosin [61], which is a known target of vertebrate CELF1 [16]. lev-11 encodes multiple isoforms regulated in a tissue-specific manner $[62,63]$.

lev-11 exons 8 and 15 were significantly overrepresented in etr-1(lq61) muscle compared to wild-type muscle (Fig. 8A). IGV-Sashimi splice junction analysis revealed that $l e v-11$ exons 8 and 15 were included in etr1(lq61) muscle and largely excluded in wild-type muscle (Fig. 8B). These results suggest that ETR-1 proteins with exon 8 controlled alternative splicing of $l e v-11$ in muscle cells, most notably removing exons 8 and 15 from lev-11 transcripts.

lev-11 RNAi resulted in AQR not migrating the full distance anteriorly, a phenotype also observed in lev11 mutants (Table 3 and Fig. $7 \mathrm{C}$ and D). The region at which AQR stopped migrating in both lev-11 RNAi and lev-11 mutants was highly stereotyped, in position 4 just posterior to the normal position. These results suggest that lev-11 might affect a specific developmental sign post rather than the general ability of $A Q R$ to migrate. In sum, our functional analysis indicates that genes with transcripts regulated by ETR-1 in muscles include those that control $A Q R$ and $P Q R$ neuron migration.

\section{Discussion}

Identification of ETR-1/CELF target genes via alternative exon usage and differential transcript abundance

CELF-family proteins regulate mRNA processing, including alternative splicing and transcript stability (reviewed in [16]). The etr-1(lq61) mutation presented a unique opportunity to determine CELF target genes in a specific tissue, body wall muscles of $C$. elegans. First, lq61 corresponds to a premature stop codon in an alternatively spliced exon affecting only a subset of etr-1 transcripts, and thus did not cause embryonic lethality [20]. Because etr-1(lq61) animals are viable, it was possible to isolate body wall muscle cells and their transcripts at a specific timepoint in L1 animals when Q neuroblasts are migrating. Second, etr-1(lq61) caused defects in AQR and PQR neuron migration in a cell-non-autonomous manner [20]. Thus, ETR-1 targets might be involved in producing a guidance signal for neurons from body wall muscle cells. Such an interaction might be conserved in vertebrate CELF function.

Two algorithms were used to compare differences in the transcriptomes of wild-type and etr-1(lq61) animals. DEXseq was used to assay alternative exon representation, and DEseq2 was used to assay differential transcript representation and splice junction usage. This analysis identified 244 genes with significant differential exon representation, and 1180 transcripts, corresponding to 971 genes, that were differentially represented. 102 genes were identified in both analyses, suggesting that these are not discrete categories and that exon abundance can affect predicted transcript abundance and vice versa. Our results suggest that a gene-by-gene approach using output from DEXseq, DEseq2, and IGV-Sashimi gives

(See figure on next page.)

Fig. 5 Gene ontology (GO) term analysis of genes with differential transcript representation in etr-1(lq61). Figure is as described in Fig. 3. The six most significant $\mathrm{GO}$ terms ( $q \leq 0.05)$ for three categories (biological process, cellular component, and molecular function) are shown. A All genes with transcripts affected by etr-1 (lq61). B Genes with underrepresented transcripts. C Genes with overrepresented transcripts. D Genes with both underrepresented and overrepresented transcripts. The entire GO term analysis is in Supplemental File 5 

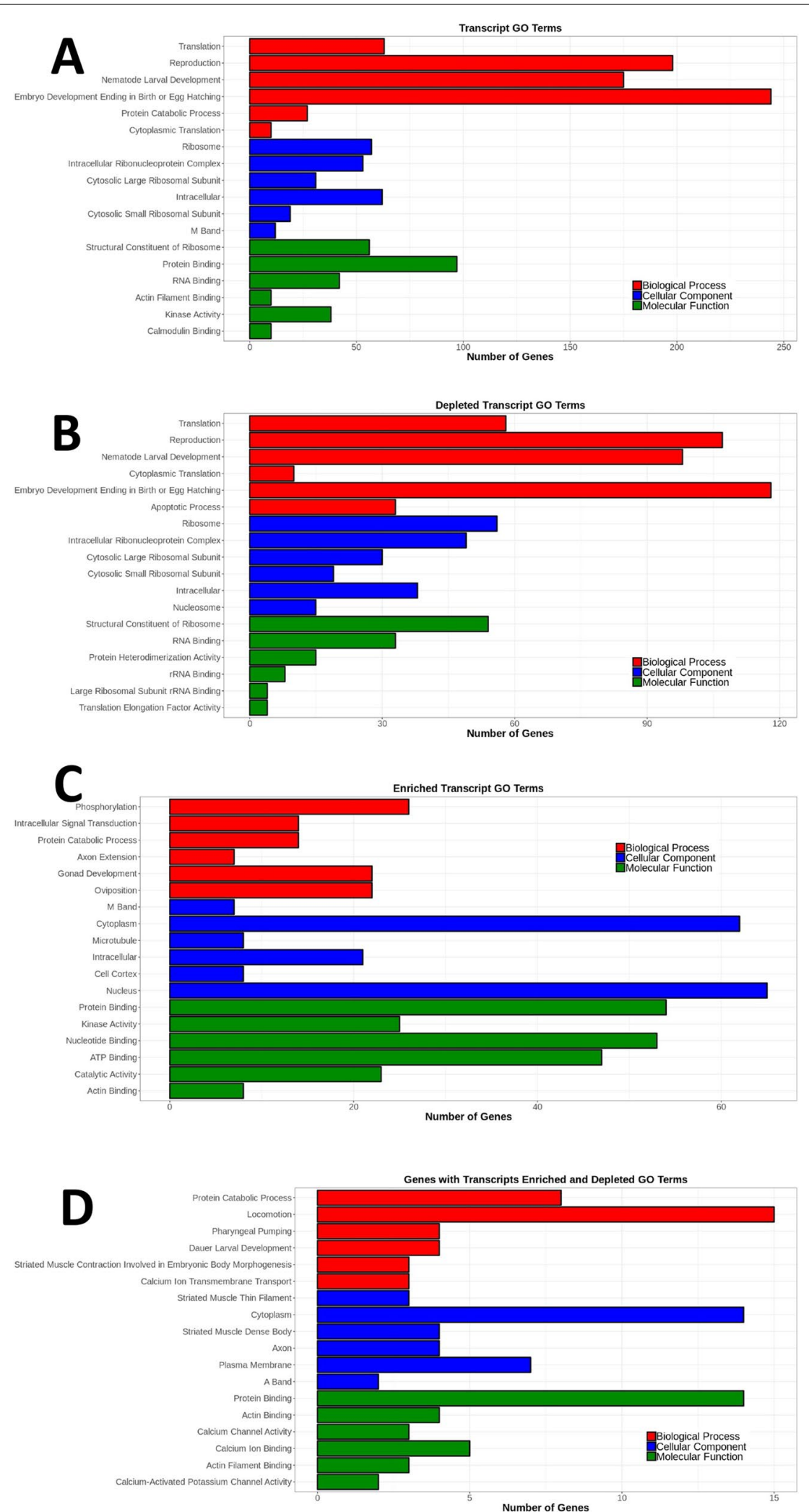

Fig. 5 (See legend on previous page.) 
Table 2 Genes with both exon representation and transcript accumulation affected by etr-1(lq61)

\begin{tabular}{|c|c|c|c|}
\hline atn-1 & $m r p-1$ & unc-73 & cpna-2 \\
\hline$a v r-14$ & $n h r-61$ & unc-87 & $w h t-1$ \\
\hline$c c r-4$ & $n t l-2$ & unc-89 & $f \mid n-2$ \\
\hline ced-1 & num-1 & unc-96 & $c r h-2$ \\
\hline ctn-1 & ketn-1 & ver-1 & C27H5.2 \\
\hline$d e b-1$ & ret-1 & $z m p-1$ & $p x \mid-1$ \\
\hline$d u r-1$ & $r m e-1$ & $a l x-1$ & $\operatorname{tag}-250$ \\
\hline alp-1 & rpl-19 & C06B3.6 & $\operatorname{acs}-17$ \\
\hline egl-10 & sli-1 & ceh-79 & fhod-1 \\
\hline etr-1 & slo-1 & $t b c-19$ & C56G2.9 \\
\hline$g c y-31$ & srp-7 & pde-3 & $F 13 C 5.5$ \\
\hline gld -2 & $\lim -9$ & shw-1 & F20A1.6 \\
\hline gon-1 & $t t n-1$ & $F 23 B 12.7$ & $\operatorname{dip}-2$ \\
\hline$h s p-4$ & $d g k-4$ & pole-1 & F44E7.4 \\
\hline kin-1 & cpna-1 & $m / c d-1$ & F46F11.1 \\
\hline lea-1 & tns-1 & F4OF8.5 & F48E3.8 \\
\hline lec-2 & $\operatorname{tax}-6$ & F43G9.12 & K09F6.10 \\
\hline lec-3 & $t m d-2$ & F49E2.5 & $\operatorname{tr} x-3$ \\
\hline lev-11 & $t n t-3$ & yif-1 & М01Н9.3 \\
\hline $\operatorname{lin}-45$ & $t s p-17$ & magi-1 & R11G1.6 \\
\hline lip-1 & unc-32 & nep-21 & T23E7.2 \\
\hline Ism-5 & unc-36 & T28F4.5 & zig-11 \\
\hline mai-1 & unc-52 & grdn-1 & $f \mid n-1$ \\
\hline mel-11 & unc-64 & Y105E8A.25 & him-19 \\
\hline$m / p-1$ & unc- 68 & nit-1 & ZC449.5 \\
\hline
\end{tabular}

a clear picture of the effects on transcript structure and abundance.

It is likely that other ETR-1 targets were not identified in this analysis, as only a subset of etr-1 isoforms containing exon 8 are affected by etr-1(lq61). Exon 8 encodes a polyglutamine-rich region [20], which is thought to
ZK370.4

$n s y-7$ 

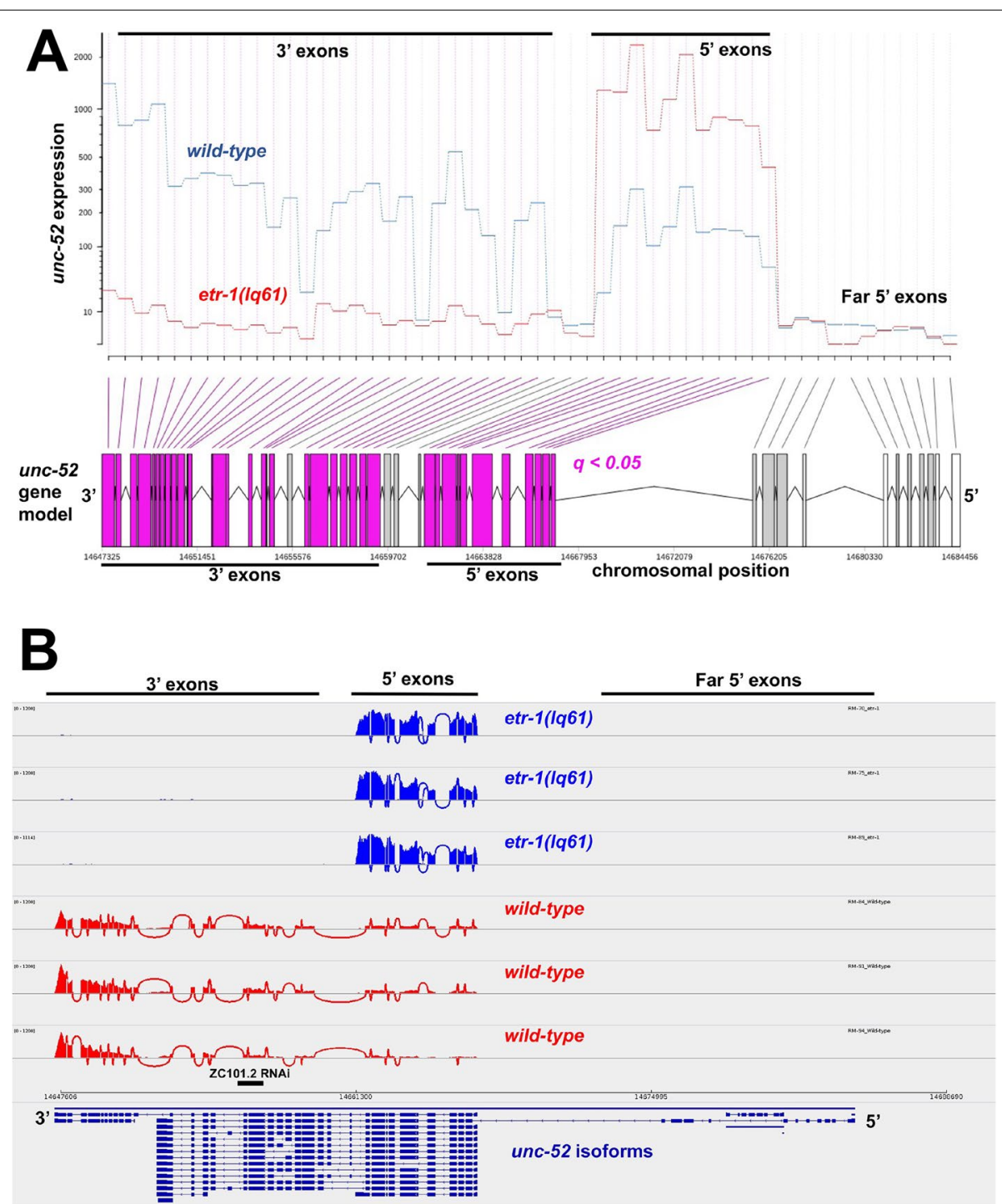

Fig. 6. etr-1(lq61) affects unc-52 exon usage. A DEXSeq output comparing etr-1(lq61) muscle to wild-type muscle as described in Fig. 2. In this plot, the $5^{\prime}$ end of the gene is to the right due to the gene being on the minus genomic strand in the .gtf file. B An IGV-Sashimi plot comparing unc-52 exon usage in etr-1(Iq61) muscle cells and wild-type muscles. The blue peaks represent etr-1(lq61) splice junctions in muscle cells in three independent biological replicates, and the red peaks represent splice junctions in wild-type muscles in three independent biological replicates

ETR-1 target genes include those involved in myofilament lattice assembly and function, muscle cell physiology, and translation

GO term analysis of ETR-1 target genes identified broad effects on the muscle cell transcriptome. The most significant GO terms described genes involved in myofilament lattice structure, assembly, and attachment were identified, along with genes involved in muscle cell physiology. This is consistent with the Pat phenotype of etr-1 RNAi [19] and with the known effects of CELF family members in muscle cell physiology. GO terms involving germ cell and reproduction were also defined, consistent with a known role of etr-1 in germ cell corpse apoptosis [26]. These genes might have conserved functions in the germline and in muscle.

Strikingly, the most significantly enriched GO terms describing genes with underrepresented transcripts in etr-1(lq61) involved translation and ribosomal function. Translation and ribosome GO terms were largely absent from the most significant GO terms describing genes with overrepresented transcripts and alternative exon usage, suggesting translation and ribosome GO terms were specifically associated with genes that require ETR-1 for transcript accumulation. This also suggests a general 

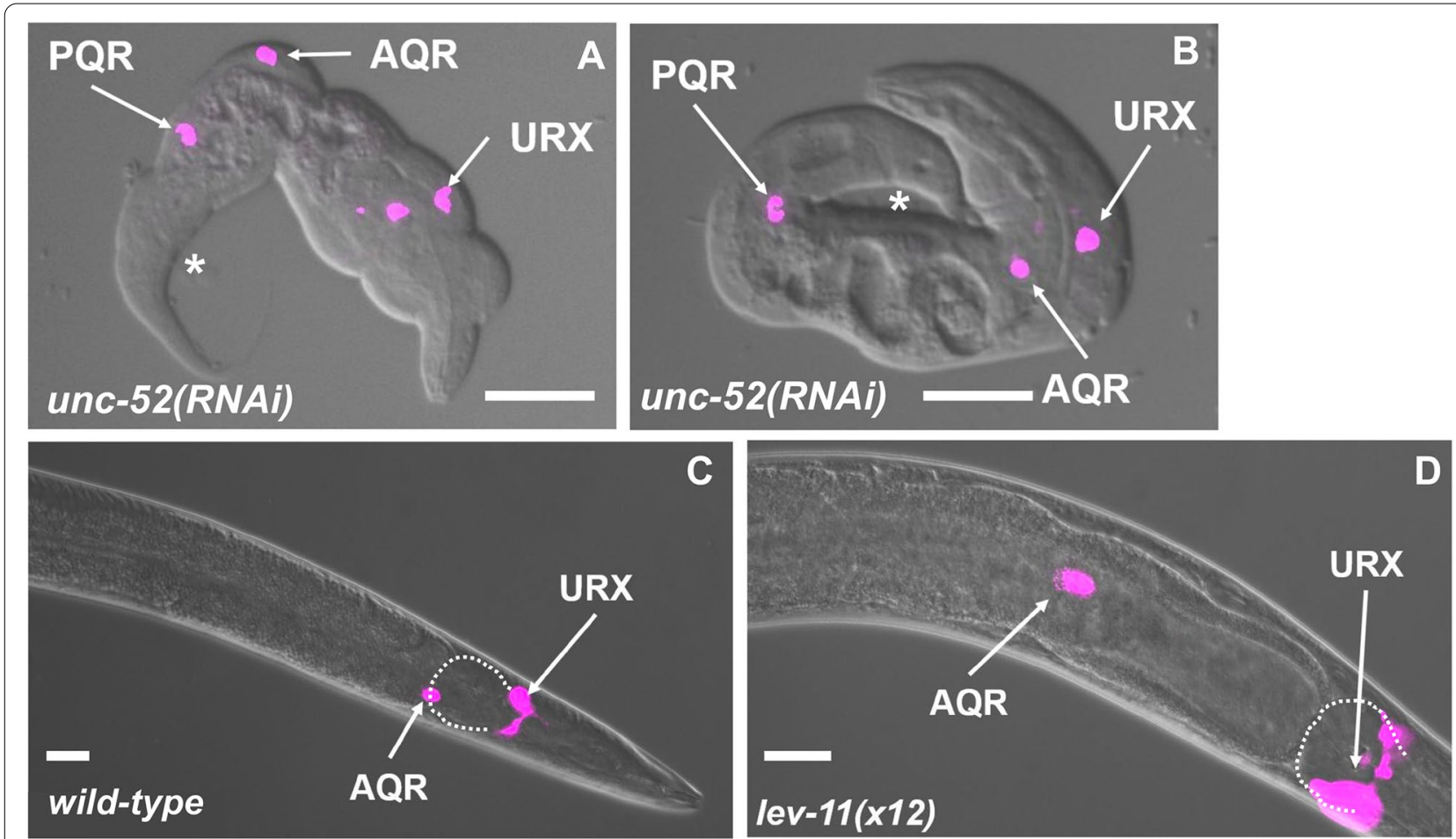

Fig. 7 AQR and PQR defects in RNAi knockdown of unc-52 and lev-11. Micrographs of merged DIC and fluorescence images are shown, with gcy-32:: $\mathrm{Cfp}$ expression in AQR and PQR (magenta). Two representative examples of paralyzed arrested at two-fold stage (Pat) unc-52(RNAi) animals are shown. The anus is indicated by an asterisk. A An unc-52(RNAi) animal displayed misplaced AQR and PQR. B An unc-52(RNAi) animal displayed a misplaced $P Q R$, with $A Q R$ in the normal position. C The wild-type position of AQR just posterior to the posterior pharyngeal bulb (outlined with a dashed white line). B AQR position in a lev-11(× 12) mutant. AQR was displaced posteriorly approximately $50 \mu \mathrm{m}$ from the posterior pharyngeal bulb. Scale bars represent $10 \mu \mathrm{m}$

impairment of translation in etr-1(lq61) muscles. siRNA knock-down of CELF1 in chicken cardiomyocytes also led to decreased expression of coding sequences with GO terms associated with translation and ribosomal function [17]. These parallel findings point to a potentially ancient evolutionary origin of CELF-family targets. Indeed, known targets of CELF molecules include tropomyosin, actinin, and troponin [16, 65]. Our analysis identified lev-11/tropomyosin, atn-1/actinin, and tht-3/troponin as ETR-1 targets with differential alternative splicing events. Depletion of etr-1 results in increased apoptotic bodies in the gonad, dependent upon the CED-1 cell corpse scavenger receptor [41]. Our analysis in muscles revealed a significant increase in the ced-1 transcript in etr-1(lq61) $\left(\log _{2}\right.$ fold change 1.58, $q=0.002$ ), suggesting that the interaction with CED-1 might be conserved in muscles.

Other known CELF1 targets were not identified in our analysis. For example, MTMR1 and MTMR3, encoding myotubularin-related proteins, are alternatively spliced by CELF1 during mouse heart development $[12,13,66]$. However, we did not detect significant differences in exon or transcript abundance of the related C. elegans genes, $m t m-1$ and $m t m-3$. Possibly, $m t m-1$ and $m t m-3$ are not regulated by ETR-1 isoforms that contain exon 8 . Alternately, ETR-1 might not regulate $\mathrm{mtm}-1$ and $\mathrm{mtm}$ 3 in body wall muscle in C. elegans, as these genes were identified as CELF1 targets in cardiomyocytes [12, 13].

\section{Targets shared between ETR-1 and UNC-75}

The CELF family of proteins contains six members in mouse and humans [16]. In C. elegans there are two members, ETR-1, similar to CELF1-2, and UNC-75, similar to CELF3-6. unc-75 function is predominantly restricted to the nervous system [21-25]. Targets of UNC-75 in the nervous system include unc-32, which encodes for the $\alpha$ subunit of the $\mathrm{V}_{0}$ complex of vacuolar-type $\mathrm{H}^{+}$-ATPases [24], and unc-64/syntaxin [22, 23]. Both $u n c-32$ and $u n c-64$ were identified as ETR-1 targets in body wall muscle cells in our analysis. This finding suggests that ETR-1 and UNC-75 might regulate common targets in a tissue-specific manner (i.e. ETR-1 in muscle, and UNC-75 in neurons).

\section{ETR-1 targets control AQR and PQR neuronal migration}

Previous studies showed that ETR-1 acts cell-nonautonomously in muscle to control AQR and PQR 
Table 3 Candidate gene AQR and PQR migration defects using RNAi unless otherwise noted

\begin{tabular}{|c|c|c|c|c|c|c|c|c|c|c|}
\hline \multirow[b]{2}{*}{ Genotype } & \multicolumn{5}{|c|}{ AQR } & \multicolumn{5}{|c|}{ PQR } \\
\hline & 1 & 2 & 3 & 4 & 5 & 1 & 2 & 3 & 4 & 5 \\
\hline wild-type & 100 & 0 & 0 & 0 & 0 & 0 & 0 & 0 & 0 & 100 \\
\hline \multicolumn{11}{|c|}{ Genes with directional migration defects } \\
\hline skn-1 & 97 & 1 & 0 & 0 & 2 & 2 & 0 & 0 & 0 & 98 \\
\hline unc-52 & 79 & 5 & 10 & 4 & 2 & 13 & 4 & 5 & 32 & 46 \\
\hline unc-71 & 91 & 4 & 0 & 0 & 5 & 5 & 0 & 0 & 0 & 95 \\
\hline \multicolumn{11}{|c|}{ Genes with defects in the ability to migrate (>10\% defects) } \\
\hline ani-1 & 89 & 11 & 0 & 0 & 0 & 0 & 0 & 0 & 2 & 98 \\
\hline$h m g-5$ & 80 & 20 & 0 & 0 & 0 & 0 & 0 & 0 & 0 & 100 \\
\hline lea-1 & 89 & 11 & 0 & 0 & 0 & 0 & 0 & 0 & 0 & 100 \\
\hline lev-11 & 94 & 6 & 0 & 0 & 0 & 0 & 0 & 0 & 1 & 99 \\
\hline lev-11(x1) & 15 & 85 & 0 & 0 & 0 & 0 & 0 & 0 & 0 & 100 \\
\hline lev-11(x12) ${ }^{1}$ & 0 & 100 & 0 & 0 & 0 & 0 & 0 & 0 & 3 & 97 \\
\hline $\operatorname{lin}-45$ & 85 & 15 & 0 & 0 & 0 & 0 & 0 & 0 & 0 & 100 \\
\hline mig-22 & 88 & 12 & 0 & 0 & 0 & 0 & 0 & 0 & 3 & 97 \\
\hline noca-1 & 87 & 12 & 1 & 0 & 0 & 0 & 0 & 0 & 0 & 100 \\
\hline sop-2 & 87 & 12 & 1 & 0 & 0 & 0 & 0 & 0 & 1 & 99 \\
\hline unc-89 & 89 & 11 & 0 & 0 & 0 & 0 & 0 & 0 & 0 & 100 \\
\hline ver-1 & 78 & 22 & 0 & 0 & 0 & 0 & 0 & 0 & 0 & 100 \\
\hline \multicolumn{11}{|c|}{ Genes with weak or no migration defects ( $<10 \%$ defects) } \\
\hline atn-1 & 92 & 8 & 0 & 0 & 0 & 0 & 0 & 0 & 0 & 100 \\
\hline ced-1 & 98 & 2 & 0 & 0 & 0 & 0 & 0 & 0 & 1 & 99 \\
\hline ceh-79 & 92 & 8 & 0 & 0 & 0 & 0 & 0 & 0 & 0 & 100 \\
\hline kin-1 & 100 & 0 & 0 & 0 & 0 & 0 & 0 & 0 & 1 & 99 \\
\hline mel-11 & 94 & 6 & 0 & 0 & 0 & 0 & 0 & 0 & 0 & 100 \\
\hline tnt-3 & 95 & 5 & 0 & 0 & 0 & 0 & 0 & 0 & 0 & 100 \\
\hline
\end{tabular}

neuronal migration [20]. ETR-1 might be involved in the generation of a signal from body wall muscles that controls $A Q R$ and $P Q R$ migration. Body wall muscle is a known source of cues that control $Q$ neuroblast lineage migration. SPON-1/F-spondin from posterior body wall muscles is required for robust $A Q R$ and PQR migration [40]. Furthermore, the NFM-1/NF-2 molecule acts non-autonomously, possibly in muscles, to control $\mathrm{Q}$ neuroblast protrusion and migration [39].

ETR-1 targets analyzed with the strongest effects on $\mathrm{AQR}$ and PQR migration include $s k n-1, u n c-71, u n c-52$, and $l e v-11$. The ADAM metalloprotease UNC-71 was previously implicated in anterior QR migrations [50,51], and our results suggest that UNC-71 also controls directional PQR migration. SKN-1, UNC-52, and LEV-11 have not been previously implicated in $\mathrm{Q}$ lineage migration. The NFE2L1 transcriptional regulator SKN-1 is a known regulator of muscle differentiation $[52,53]$. While RNAi knockdown of these genes in all tissues by feeding RNAi caused $A Q R$ and $P Q R$ migration defects, further studies will be required to show that ETR-1 regulation of these genes in muscle cells is relevant to $A Q R$ and $P Q R$ migration (i.e. these genes might act in other cells besides muscles to control $\mathrm{AQR}$ and $\mathrm{PQR}$ migration).

\section{unc-52/Perlecan is a target of etr-1 and is required for $A Q R$ and $P Q R$ migration}

unc-52 encodes the basement membrane heparan sulfate proteoglycan Perlecan and is expressed in many different cell types, including the muscle cells $[49,58,67]$. unc-52 has been shown to control the migrations of the distal tip cells [68]. The heparan sulfate epimerase HSE-5 has been shown to control $Q$ neuroblast migration $[69,70]$, but loss of no single HSPG or in double mutant combination has been shown to affect $\mathrm{Q}$ migrations, including hypomorphic unc-52 alleles [70].

unc-52 was identified as a target of ETR-1 in muscles, with the $3^{\prime}$ exons of unc-52 underrepresented in etr1(lq61). This finding suggests that ETR-1 with exon 8 is required for the accumulation of the long unc-52 isoforms with the $3^{\prime}$ exons. Depletion of unc-52 by RNAi resulted in embryonic lethality and severe $A Q R$ and $P Q R$ migration defects, including directional defects similar to hse-5. This finding suggests that UNC-52 might be the HSPG through which HSE-5 is controlling Q migration. 

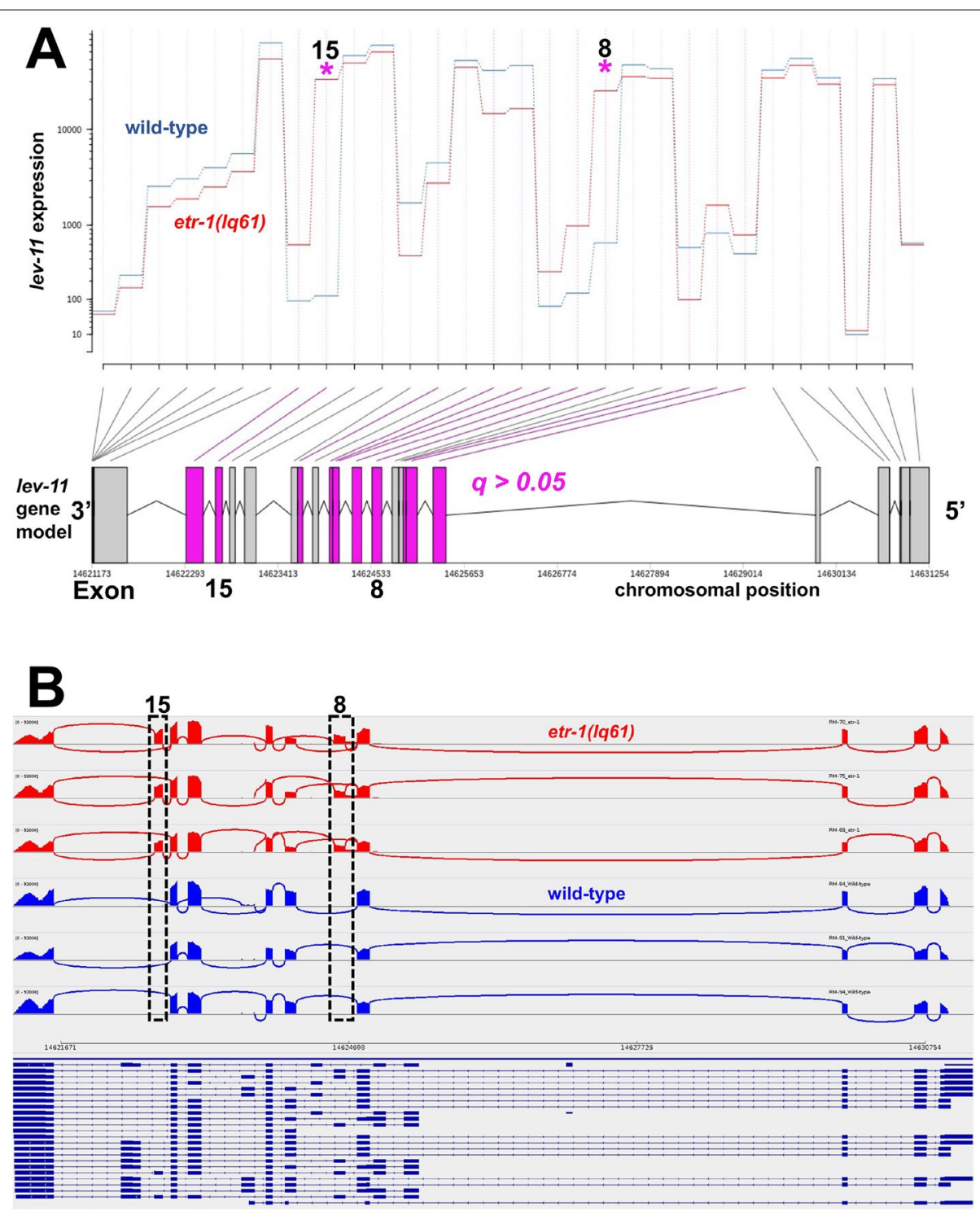

Fig. 8. etr-1(lq61) affects lev-11 exon usage. A DEXSeq output for lev-11 comparing exon usage levels between etr-1(lq61) muscle and wild-type muscle, as described in Fig. 2. B IGV-Sashimi plot of lev-11 splice junctions in wild-type and etr-1(lq61), as described in Fig. 6

Both unc-52 and hse-5 mutants display defects in AQR and $\mathrm{PQR}$ direction of migration, suggesting that UNC52 is not merely a substrate for cell migration but rather provides directional information.

Our analysis showed that unc-52 isoforms containing the $3^{\prime}$ exons were strongly underrepresented in etr1(lq61) muscle, with the shorter ZC101.2b.1 transcript the predominant unc-52 mRNA. The unc-52 RNAi clone we used is located in these $3^{\prime}$ exons, suggesting that the long isoforms with the $3^{\prime}$ exons are important for AQR and PQR migration. The $3^{\prime}$ exons mainly encode for Immunoglobulin family domains, some of which are alternatively spliced in the epidermis by other factors, MEC-8 and CCAR-1 [56-58]. RNAi of $u n c-52$ resulted in embryonic lethality, yet etr-1(lq61), which nearly eliminates transcripts with the $3^{\prime}$ exons in muscles, is viable. This is likely due to unc-52 function in other tissues not regulated by etr-1 (i.e. epidermis). Indeed, mec- 8 function in epidermis is sufficient to rescue mec-8; unc-52 synthetic lethality and the Pat phenotype [58]. This is consistent with the idea that unc-52 long isoforms in muscle are not required for embryonic viability but are required for $A Q R$ and $P Q R$ migration. 


\section{lev-11 is alternatively spliced by etr-1 and is required} for $A Q R$ and $P Q R$ migration

We showed that ETR-1 exon 8 isoforms are required for alternative splicing of $l e v-11$ transcripts in muscle by the removal of exons 8 and 15 (Fig. 8B). It was shown previously that CELF proteins regulate the alternative splicing of tropomyosin in chicken muscle development [71]. Isoforms of $l e v-11$ are expressed in a tissue-specific manner [62]. lev-11 isoforms without exons 8 and 15 are expressed robustly in wild-type body wall muscle cells [62] (Fig. 8B), whereas those containing lev-11 exons 8 and 15 are expressed in pharyngeal muscle and the excretory cell [62]. Our results suggest that ETR-1 with exon 8 is required to exclude exons 8 and 15 from lev-11 transcripts in body wall muscle cells. Possibly, lev-11 isoforms lacking exons 8 and 15 are optimized for wall body muscle structure or function.

Knockdown of lev-11 by either RNAi or in lev-11 mutants resulted in AQR migration defects, with most AQR cells stopping approximately $50 \mu \mathrm{m}$ posterior to its normal position near the posterior pharyngeal bulb. This characteristic stopping point suggests that lev-11 affects a developmental guidepost rather than generally affecting the ability of AQR to migrate, which results in stoppages of AQR along anterior body in positions 2, 3, and 4 (e.g. epi-1/laminin mutants) [72]. Two-hybrid studies suggest that LEV-11 physically interacts with MIG-14/ Wntless [73], which is involved involved in Wnt secretion [74-76]. Mutation of mig-14 results in Q neuroblast migration defects $[37,77,78]$, including AQR and PQR directional defects [38]. etr-1 interacts genetically with Wnt mutations to control AQR and PQR migration, either directly or through a parallel pathway [20]. Expression of three Wnt genes is significantly increased in body wall muscle (Supplemental File 1): egl-20 ( $\log 2-$ fold change $=2.96), c w n-1(\log 2$-fold change $=2.74)$, and $c w n-2(\log 2$-fold change $=1.02)$, but their exon use and transcript levels are not affected by etr-1(lq61). Intriguingly, $c w n-1$ mutants display posteriorly-displaced AQR similar to but weaker than lev-11 mutants [38]. It is plausible that etr-1 regulates the splicing of $l e v-11$ in muscle to control an interaction with MIG-14. Disrupting this interaction might result in improper production of a Wnt signal, causing AQR and PQR migration defects. Further studies will be aimed at exploring this mechanism.

\section{Linking the effects of etr-1(Iq61) on the muscle cell transcriptome to mutant phenotypes}

Here we define transcripts in body wall muscle cells that are affected by mutation of the CELF1 family member etr-1. The etr-1(lq61) mutation selectively disrupts isoforms that include exon 8, leaving other ETR-1 isoforms intact. Thus, this set of body muscle transcripts include only those affected by ETR-1 with exon 8. etr-1(lq61) affected splicing and accumulation of transcripts of genes involved in a broad spectrum of muscle cell function including myofilament structure and attachment and physiology. Strikingly, genes involved in translation and ribosomal function were abundant among genes with transcripts underrepresented in etr-1(lq61). siRNA knock-down of CELF1 in chicken cardiomyocytes also led to a decrease in genes with GO terms associated with translation and ribosomal function [17], suggesting a deep evolutionary conservation of CELF1 target genes. Indeed, lev-11/tropomyosin, atn-1/actinin, and tht-3/troponin were also conserved targets in C. elegans and vertebrates [16, 65].

How do these broad effects on the muscle transcriptome result in etr-1(lq61) mutant phenotypes, including $A Q R$ and $P Q R$ migration? We demonstrate that genes identified here include those that control AQR and PQR migration, such as $u n c-52$ and $l e v-11$. What is less clear is how the etr-1-specific effects on these transcripts in muscle cells contributes to $A Q R$ and $P Q R$ migration defects. It seems unlikely that the AQR and PQR defects in etr1(lq61) result from disruption of one or a small handful of genes. Rather, more likely is that subtle effects on multiple genes controlling AQR and PQR migration contribute to the phenotype, such as $u n c-52, l e v-11$, and others. Future studies will be aimed at addressing this question.

\section{Materials and methods}

\section{Strains and genetics}

C. elegans strains were was cultured using standard methods at $20^{\circ} \mathrm{C}$. Wild-type strain was N2 Bristol. Alleles used were: LGII: etr-1(lq61), lqIs244[Pgcy-32::cfp], lex-11( $\times 1$ and $\times 12)$. LG V: lqIs58[Pgcy-32::cfp]. LG unknown: ccIs4521[myo-3p::GFP::LacZ::NLS + (pSAK4) myo-3p::mitochondrial GFP+dpy-20(+)] [79].

\section{Larval disruption for cell sorting}

For body wall muscle cell sorting, the myo-3::gfp transgene ccIs4521 was used, in wild-type and etr-1(lq61) backgrounds. C. elegans strains were grown on twenty $\times 150 \mathrm{~mm} 8 \mathrm{P}$ nutrient agar plates (for $1 \mathrm{l}: 20 \mathrm{~g}$ bactopeptone, $3 \mathrm{~g} \mathrm{NaCl}, 25 \mathrm{~g}$ agar) with $E$. coli strain NA22, which produces a thick bacterial lawn. Early L1 animals were synchronized by first collecting embryos using bleach (hypochlorite) treatment of gravid adult hermaphrodites, and allowing the embryos to hatch overnight into sterile $\mathrm{M} 9$ medium at $20^{\circ} \mathrm{C}$. This produced approximately 3 million starved, synchronized early L1 larvae. The Q neuroblasts of starved L1s were variably arrested in their early development, from initial migration to the first division after migration (data not shown). Preparations of dissociated larval cells were generated as previously described 
using SDS-DTT and Pronase treatment coupled with mechanical disruption using a pipet $[42,43]$.

\section{FACS analysis}

Fluorescence activated cell sorting of myo-3::GFPexpressing body wall muscle cells was performed as previously described using a BD FACSAria with a $70 \mu \mathrm{m}$ diameter nozzle [42, 43]. DAPI was added to mark dead or damaged cells which were excluded from each sample of viable myo-3::GFP labeled muscle cells. Profiles of GFP strains were compared to a non-transgene-bearing N2 standard to exclude auto-fluorescent cells. Cells were sorted into Trizol LS. RNA was extracted from the aqueous phase using 5PRIME phase lock gel heavy tubes and purified on a spin column (Zymo, R1013). 30,000-50,000 FACS-sorted cells from each experiment, yielded 5-100 ng total RNA which was used in RNA seq library preparation. Three independent replicate samples were obtained for both wild-type and etr-1(lq61) mutant animals.

\section{mRNA library preparation and sequencing methods}

For an all-cells control group, total RNA was isolated from an aliquot of $\sim 25,000 \mathrm{~L} 1$ larvae set aside before cell dissociation and FACS for each one of the samples. L1 larvae were quickly frozen in liquid nitrogen in a mortar and pestle and ground to powder. Trizol LS was added to the powdered animals for RNA extraction. Muscle FACS and whole L1 RNA extraction resulted in twelve RNA samples representing three biological replicates of $\mathrm{N} 2$ and etr-1(lq61) sorted muscles and three biological replicates of $\mathrm{N} 2$ and etr-1(lq61) whole L1 larvae all-cell controls. Quality of total RNA samples was ensured using an Agilent TapeStation, and quantity was determined using Qubit fluorimetry. Sequencing libraries were constructed with 5-100 ng of total RNA using the NEBNext Ultra II Directional RNA Library Kit for Illumina. The sequencing library construction process aimed for 300-bp inserts and included mRNA purification with poly-A beads, fragmentation, strand specific cDNA synthesis, end repair, $3^{\prime}$ end adenylation, adapter ligation, and PCR amplification. The constructed sequencing libraries were validated and quantified with Qubit and TapeStation assays. Each library indexed and sequenced in multiplex on the Illumina NextSeq550 system, generating paired-end, 150-base sequence reads from the libraries. Between 33 million and 39 million reads were generated for each of the 12 samples. Base calling was carried out by the Nextseq550 instrument Real Time Analysis (RTA) software. The base call files (bcl files) were demultiplexed and converted to compressed FASTQ files by bcl2fastq2.

\section{Data availability statement}

All data are represented in this manuscript and all strains and reagents are available upon request. FASTQ files for this project are available in the Sequence Read Archive (SRA) under project number PRJNA733501. Computational code can be found in Supplemental Materials.

\section{RNA-Seq analysis}

The C. elegans reference genome was downloaded from the following URL: //hgdownload.cse.ucsc.edu/goldenPath/ce11/bigZips/chromFa.tar.gz. Quality control of reads was performed using FastQC (version 0.11.5) and fastp (options: --detect_adapter_for_pe -length_required 75 -trim_front1 10 -trim_front2 10 -cut_mean_quality 25 -cut_window_quality 5 -cut_tail, version 0.19.8) [80]. Splicesite and exon models were built using the C. elegans release 11 GTF file and HISAT2 with default settings (hisat2_extract_splice_sites.py and hisat2_extract_exons. py). Reads were aligned to the genome using HISAT2 with default settings (version 2.1.0) [81]. Samtools (version 1.7) [82] was used to convert SAM files to BAM files, and to sort the BAM files for downstream analysis. Read counts were assembled using stringTie (version 1.3.5) [83, 84], and FeatureCounts (version 1.6.0) [85]. We used the accompanying Python script from stringTie to prepare the output specifically for use in DESeq2 [86].

\section{Differential exon usage analysis}

Alternative splicing differences were determined by quantifying differential exon usage using the Bioconductor package DEXSeq (version 1.30.0) [46] in R. Expression profiles were built using FeatureCounts [85]. Exons that showed differential expression with an adjusted $p$ value false discovery rate less than 0.05 were considered differentially expressed in our analysis. Briefly, in DEXseq, exon or exon bin representation is normalized against the total number of aligned reads in the sample using the estimateSizeFactors function, and a $X^{2}$ test with dispersion estimates and corrected for multiple testing is used to derive a $q$ value significance.

\section{Differential transcript expression analysis}

We examined all cells versus muscle cells, and wildtype versus etr-1(lq61) muscle cells in separate analyses. Differential transcript expression was tested using the Bioconductor package DESeq2 (version 1.24.0) in R. Expression profiles were prepared using stringTie [83, 84], and the accompanying Python script was used to 
prepare a table for DESeq2. For both analyses, transcripts with a false-discovery rate adjusted $p$-value less than 0.05 were considered significantly differentially expressed. A similar statistical framework is used in DEseq2 as is used in DEXseq described above ( $\chi^{2}$ with dispersion estimates and correction for multiple testing). Preparation of tables and graphs was carried out in R [87].

\section{Gene ontology analysis}

Analysis was done using the Database for Annotation, Visualization and Integrated Discovery (DAVID: https:// david.ncifcrf.gov/) [47, 48]. Alternative exon usage and alternative transcript expression were analyzed for biological process, cellular component, and molecular function separately. Gene lists were analyzed for terms that were significantly enriched at $q>0.05$ against all $C$. elegans genes. The complete GO term list for each separate test are in Supplementary files 3 and 5. Graphs were prepared in $\mathrm{R}$ [87].

\section{Sashimi plots}

Sashimi plots were created using the Integrative Genomics Viewer (IGV), version 2.5.0 [88, 89]. Indexes to visualize the splice junctions on IGV were built using the samtools (version 1.7.0) index function [82].

\section{RNA-mediated gene interference (RNAi)}

RNAi was administered via feeding, following standard protocols and clones from the Source BioScience library (Nottingham UK) [90, 91]. (Kamath et al., 2003). For each RNAi experiment we grew wild-type animals expressing Pgcy-32::cfp to visualize AQR and $\mathrm{PQR}$ on RNAi bacteria [92]. For each independent set of RNAi experiments, ceh-20(RNAi) was used as a positive control, as RNAi of ceh-20 results in robust $A Q R$ and $P Q R$ defects [92].

\section{Scoring $A Q R$ and $P Q R$ migration defects}

$\mathrm{AQR}$ and $\mathrm{PQR}$ migration was scored using $g c y$-32::cfp as previously reported [20,30,31]. Briefly, L4 animals were collected, mounted onto a $2 \%$ agar pad and immobilized by $5 \mathrm{mM}$ of $\mathrm{NaN}_{3}$. Five positions along the length of the animal were noted. Position 1 is the normal $A Q R$ position in the head just posterior to the posterior pharyngeal bulb; position 2 is posterior to the normal position but anterior to the vulva, position 3 is proximal to the vulva both anteriorly and posteriorly, position 4 is the normal birthplace of QR and $\mathrm{QL}$, and position 5 is the normal location of PQR, just posterior to the anus. 100 animals were scored for each RNAi clone.

\section{Supplementary Information}

The online version contains supplementary material available at https://doi. org/10.1186/s12864-021-08217-6.

Additional file 1.

Additional file 2.

Additional file 3.

Additional file 4.

Additional file 5.

Additional file 6 .

Additional file 7.

Additional file 8.

Additional file 9.

Acknowledgements

The authors thank the members of the Lundquist and Ackley labs for discussion and E. Struckhoff and Z. Grant for technical assistance. Some strains were provided by the CGC, which is funded by $\mathrm{NIH}$ Office of research infrastructure programs (P40OD010440). Sequencing was conducted th the KU genome sequencing Core supported by the National Institute of General Medical Sciences (P20GM103638), and the Kansas infrastructure Netrwork of biomedical research excellence (P20GM103418) for computational support.

\section{Authors' contributions}

M.E.O. designed and conducted experiments, analyzed data, made figures and tables, and wrote the manuscript. R.M.M. conducted experiments. R.L.U. analyzed data and edited the manuscript. D.M.M. designed experiments and edited the manuscript. E.A.L. designed and conducted experiments, analyzed data, and edited the manuscript. The author(s) read and approved the final manuscript.

\section{Funding}

This work was supported by National Institute of Health projects R21NS100483 to E.A.L. and D.M.M.; R01NS106951 and R01NS10054 to D.M.M.; and R01Al139154 to R.L..

\section{Availability of data and materials}

All data are represented in this manuscript and all strains and reagents are available upon request from the corresponding author (erikl@ku.edu). FASTQ files for this project are available in the Sequence Read Archive (SRA) under project number PRJNA733501 (https://www.ncbi.nlm.nih.gov/sra/?term= PRJNA733501). Computational code can be found in Supplemental Materials.

\section{Declarations}

Ethics approval and consent to participate NA

\section{Consent for publication}

All authors agree to publish the contents of the manuscript as presented.

\section{Competing interests}

The authors declare that there are no competing interests.

\section{Author details}

${ }_{1}^{1}$ Program in Molecular, Cellular, and Developmental Biology, Department of Molecular Biosciences, University of Kansas, Lawrence, KS 66045, USA.

${ }^{2}$ Department of Cell and Developmental Biology and Department of Biological Sciences, Vanderbilt University, Nashville, TN 37203, USA.

Received: 13 October 2021 Accepted: 23 November 2021 Published online: 06 January 2022 


\section{References}

1. Schoser B, Timchenko L. Myotonic dystrophies 1 and 2: complex diseases with complex mechanisms. Current Genomics. 2010;11(2):77-90.

2. Savkur RS, Philips AV, Cooper TA. Aberrant regulation of insulin receptor alternative splicing is associated with insulin resistance in myotonic dystrophy. Nat Genet. 2001;29(1):40-7.

3. Timchenko NA, Cai ZJ, Welm AL, Reddy S, Ashizawa T, Timchenko LT. RNA CUG repeats sequester CUGBP1 and alter protein levels and activity of CUGBP1. J Biol Chem. 2001:276(11):7820-6.

4. Kuyumcu-Martinez NM, Wang GS, Cooper TA. Increased steady-state levels of CUGBP1 in myotonic dystrophy 1 are due to PKC-mediated hyperphosphorylation. Mol Cell. 2007;28(1):68-78.

5. Ho TH, Bundman D, Armstrong DL, Cooper TA. Transgenic mice expressing CUG-BP1 reproduce splicing mis-regulation observed in myotonic dystrophy. Hum Mol Genet. 2005;14(11):1539-47.

6. Timchenko NA, Patel R, lakova P, Cai ZJ, Quan L, Timchenko LT. Overexpression of CUG triplet repeat-binding protein, CUGBP1, in mice inhibits myogenesis. J Biol Chem. 2004;279(13):13129-39.

7. Berger DS, Ladd AN. Repression of nuclear CELF activity can rescue CELF-regulated alternative splicing defects in skeletal muscle models of myotonic dystrophy. PLoS currents. 2012;4:RRN1305.

8. Li D, Bachinski LL, Roberts R. Genomic organization and isoformspecific tissue expression of human NAPOR (CUGBP2) as a candidate gene for familial arrhythmogenic right ventricular dysplasia. Genomics. 2001;74(3):396-401.

9. Wijsman EM, Pankratz ND, Choi Y, Rothstein JH, Faber KM, Cheng R, et al Genome-wide association of familial late-onset Alzheimer's disease replicates BIN1 and CLU and nominates CUGBP2 in interaction with APOE. PLoS Genet. 2011;7(2):e1001308.

10. Daughters RS, Tuttle DL, Gao W, Ikeda Y, Moseley ML, Ebner TJ, et al. RNA gain-of-function in spinocerebellar ataxia type 8. PLoS Genet. 2009;5(8):e1000600

11. Sofola OA, Jin P, Qin Y, Duan R, Liu H, de Haro $M$, et al. RNA-binding proteins hnRNP A2/B1 and CUGBP1 suppress fragile X CGG premutation repeat-induced neurodegeneration in a Drosophila model of FXTAS. Neuron. 2007;55(4):565-71.

12. Ladd AN, Taffet G, Hartley C, Kearney DL, Cooper TA. Cardiac tissue-specific repression of CELF activity disrupts alternative splicing and causes cardiomyopathy. Mol Cell Biol. 2005;25(14):6267-78.

13. Kalsotra A, Xiao X, Ward AJ, Castle JC, Johnson JM, Burge CB, et al. A postnatal switch of CELF and MBNL proteins reprograms alternative splicing in the developing heart. Proc Natl Acad Sci U S A 2008;105(51):20333-8.

14. Terenzi F, Brimacombe KR, Penn MS, Ladd AN. CELF-mediated alternative splicing is required for cardiac function during early, but not later, postnatal life. J Mol Cell Cardiol. 2009:46(3):395-404.

15. Kim G, Pai Cl, Sato K, Person MD, Nakamura A, Macdonald PM. Regionspecific activation of oskar mRNA translation by inhibition of Brunomediated repression. PLoS Genet. 2015;11(2):e1004992.

16. Dasgupta T, Ladd AN. The importance of CELF control: molecular and biological roles of the CUG-BP, Elav-like family of RNA-binding proteins. Wiley Interdisciplinary Reviews RNA. 2012;3(1):104-21.

17. Blech-Hermoni Y, Dasgupta T, Coram RJ, Ladd AN. Identification of targets of CUG-BP, Elav-like family member 1 (CELF1) regulation in embryonic heart muscle. PLoS One. 2016;11(2):e0149061.

18. Good PJ, Chen Q, Warner SJ, Herring DC. A family of human RNA-binding proteins related to the Drosophila Bruno translational regulator. J Biol Chem. 2000:275(37):28583-92.

19. Milne CA, Hodgkin J. ETR-1, a homologue of a protein linked to myotonic dystrophy, is essential for muscle development in Caenorhabditis elegans. Curr Biol. 1999;9(21):1243-6.

20. Ochs ME, Josephson MP, Lundquist EA: The Predicted RNA-Binding Protein ETR-1/CELF1 Acts in Muscles To Regulate Neuroblast Migration in Caenorhabditis elegans. G3 2020, 10(7):2365-2376.

21. Loria PM, Duke A, Rand JB, Hobert O. Two neuronal, nuclear-localized RNA binding proteins involved in synaptic transmission. Curr Biol. 2003;13(15):1317-23.

22. Chen L, Liu Z, Zhou B, Wei C, Zhou Y, Rosenfeld MG, Fu XD, Chisholm AD, Jin Y: CELF RNA binding proteins promote axon regeneration in C. elegans and mammals through alternative splicing of Syntaxins. eLife 2016, 5.
23. Norris AD, Gao S, Norris ML, Ray D, Ramani AK, Fraser AG, et al. A pair of RNA-binding proteins controls networks of splicing events contributing to specialization of neural cell types. Mol Cell. 2014;54(6):946-59.

24. Kuroyanagi $H$, Watanabe $Y$, Hagiwara M. CELF family RNA-binding protein UNC-75 regulates two sets of mutually exclusive exons of the unc-32 gene in neuron-specific manners in Caenorhabditis elegans. PLoS Genet. 2013:9(2):e1003337.

25. Kuroyanagi H, Watanabe Y, Suzuki Y, Hagiwara M. Position-dependent and neuron-specific splicing regulation by the CELF family RNAbinding protein UNC-75 in Caenorhabditis elegans. Nucleic Acids Res. 2013;41(7):4015-25.

26. Boateng R, Nguyen KCQ, Hall DH, Golden A, Allen AK. Novel functions for the RNA-binding protein ETR-1 in Caenorhabditis elegans reproduction and engulfment of germline apoptotic cell corpses. Dev Biol. 2017:429(1):306-20.

27. Middelkoop TC, Korswagen HC: Development and migration of the $C$ elegans Q neuroblasts and their descendants. WormBook 2014:1-23.

28. Chalfie M, Sulston J. Developmental genetics of the mechanosensory neurons of Caenorhabditis elegans. Dev Biol. 1981;82(2):358-70.

29. Sulston JE, Horvitz HR. Post-embryonic cell lineages of the nematode, Caenorhabditis elegans. Dev Biol. 1977;56(1):110-56.

30. Chapman JO, Li H, Lundquist EA. The MIG-15 NIK kinase acts cell-autonomously in neuroblast polarization and migration in C. elegans. Dev Biol. 2008:324(2):245-57.

31. Sundararajan L, Lundquist EA. Transmembrane proteins UNC-40/DCC, PTP-3/LAR, and MIG-21 control anterior-posterior neuroblast migration with left-right functional asymmetry in Caenorhabditis elegans. Genetics. 2012;192(4):1373-88

32. Sundararajan L, Norris ML, Schoneich S, Ackley BD, Lundquist EA. The fat-like cadherin $\mathrm{CDH}-4$ acts cell-non-autonomously in anterior-posterior neuroblast migration. Dev Biol. 2014;392(2):141-52.

33. Middelkoop TC, Williams L, Yang PT, Luchtenberg J, Betist MC, Ji N, et al. The thrombospondin repeat containing protein MIG-21 controls a left-right asymmetric Wnt signaling response in migrating C. elegans neuroblasts. Dev Biol. 2012;361(2):338-48.

34. Ebbing A, Middelkoop TC, Betist MC, Bodewes E, Korswagen HC: Partially overlapping guidance pathways focus the activity of UNC-40/DCC along the anteroposterior axis of polarizing neuroblasts. Development 2019, 146(18).

35. Honigberg L, Kenyon C. Establishment of left/right asymmetry in neuroblast migration by UNC-40/DCC, UNC-73/trio and DPY-19 proteins in C. elegans. Development. 2000;127(21):4655-68.

36. Zinovyeva AY, Yamamoto Y, Sawa H, Forrester WC. Complex network of Wnt signaling regulates neuronal migrations during Caenorhabditis elegans development. Genetics. 2008;179(3):1357-71.

37. Harterink M, Kim DH, Middelkoop TC, Doan TD, van Oudenaarden A, Korswagen HC. Neuroblast migration along the anteroposterior axis of C. elegans is controlled by opposing gradients of Wnts and a secreted frizzled-related protein. Development. 2011;138(14):2915-24

38. Josephson MP, Chai Y, Ou G, Lundquist EA: EGL-20/Wnt and MAB-5/Hox Act Sequentially to Inhibit Anterior Migration of Neuroblasts in C. elegans. PLoS One 2016, 11 (2):e0148658.

39. Josephson MP, Aliani R, Norris ML, Ochs ME, Gujar M, Lundquist EA. The Caenorhabditis elegans NF2/Merlin molecule NFM-1 nonautonomously regulates neuroblast migration and interacts genetically with the guidance Cue SLT-1/slit. Genetics. 2017;205(2):737-48.

40. Josephson MP, Miltner AM, Lundquist EA. Nonautonomous roles of MAB-5/Hox and the secreted basement membrane molecule SPON1/F-Spondin in Caenorhabditis elegans neuronal migration. Genetics. 2016;203(4):1747-62

41. Boateng R, Allen AK. New role for an old protein: an educational primer for use with "the identification of a novel mutant allele of topoisomerase II in Caenorhabditis elegans reveals a unique role in chromosome segregation during spermatogenesis". Genetics. 2018;208(1):79-88.

42. Spencer WC, McWhirter R, Miller T, Strasbourger P, Thompson O, Hillier LW, Waterston $\mathrm{RH}$, Miller DM, 3rd: Isolation of specific neurons from C. elegans larvae for gene expression profiling. PLoS One 2014, 9(11):e112102.

43. Taylor SR, Santpere G, Weinreb A, Barrett A, Reilly MB, Xu C, Varol E, Oikonomou P, Glenwinkel L, McWhirter R et al: Molecular topography of an entire nervous system. bioRxiv 2020:2020.2012.2015.422897. 
44. Fox RM, Watson JD, Von Stetina SE, McDermott J, Brodigan TM, Fukushige $\mathrm{T}$, et al. The embryonic muscle transcriptome of Caenorhabditis elegans. Genome Biol. 2007:8(9):R188.

45. Ladd AN, Charlet N, Cooper TA. The CELF family of RNA binding proteins is implicated in cell-specific and developmentally regulated alternative splicing. Mol Cell Biol. 2001;21(4):1285-96.

46. Anders S, McCarthy DJ, Chen Y, Okoniewski M, Smyth GK, Huber W, et al. Count-based differential expression analysis of RNA sequencing data using R and bioconductor. Nat Protoc. 2013;8(9):1765-86.

47. Huang da W, Sherman BT, Lempicki RA: Systematic and integrative analysis of large gene lists using DAVID bioinformatics resources. Nat Protoc 2009, 4(1):44-57.

48. Huang da W, Sherman BT, Lempicki RA: Bioinformatics enrichment tools: paths toward the comprehensive functional analysis of large gene lists. Nucleic Acids Res 2009, 37(1):1-13.

49. Rogalski TM, Williams BD, Mullen GP, Moerman DG. Products of the unc-52 gene in Caenorhabditis elegans are homologous to the core protein of the mammalian basement membrane heparan sulfate proteoglycan. Genes Dev. 1993;7(8):1471-84.

50. Masuda H, Nakamura K, Takata N, Itoh B, Hirose T, Moribe H, et al. MIG-13 controls anteroposterior cell migration by interacting with UNC-71/ADM-1 and SRC-1 in Caenorhabditis elegans. FEBS Lett. 2012;586(6):740-6.

51. Huang $X$, Huang P, Robinson MK, Stern MJ, Jin Y. UNC-71, a disintegrin and metalloprotease (ADAM) protein, regulates motor axon guidance and sex myoblast migration in C. elegans. Development. 2003;130(14):3147-61.

52. Gomes JE, Encalada SE, Swan KA, Shelton CA, Carter JC, Bowerman B. The maternal gene spn-4 encodes a predicted RRM protein required for mitotic spindle orientation and cell fate patterning in early $C$. elegans embryos. Development. 2001;128(21):4301-14.

53. Broitman-Maduro G, Maduro MF, Rothman JH. The noncanonical binding site of the MED-1 GATA factor defines differentially regulated target genes in the C. elegans mesendoderm. Dev Cell. 2005;8(3):427-33.

54. Rogalski TM, Gilchrist EJ, Mullen GP, Moerman DG. Mutations in the unc-52 gene responsible for body wall muscle defects in adult Caenorhabditis elegans are located in alternatively spliced exons. Genetics. 1995:139(1):159-69.

55. Rogalski TM, Mullen GP, Bush JA, Gilchrist EJ, Moerman DG. UNC-52/ perlecan isoform diversity and function in Caenorhabditis elegans. Biochem Soc Trans. 2001;29(Pt 2):171-6.

56. Fu R, Zhu Y, Jiang $X$, Li Y, Zhu M, Dong M, Huang Z, Wang C, Labouesse $M$, Zhang H: CCAR-1 affects hemidesmosome biogenesis by regulating unc-52/perlecan alternative splicing in the C. elegans epidermis. J Cell Sci 2018, 131(11).

57. Lundquist EA, Herman RK, Rogalski TM, Mullen GP, Moerman DG, Shaw $J E$. The mec-8 gene of $C$. elegans encodes a protein with two RNA recognition motifs and regulates alternative splicing of unc- 52 transcripts. Development. 1996;122(5):1601-10.

58. Spike CA, Davies AG, Shaw JE, Herman RK. MEC-8 regulates alternative splicing of unc-52 transcripts in C. elegans hypodermal cells. Development. 2002;129(21):4999-5008.

59. Williams BD, Waterston RH. Genes critical for muscle development and function in Caenorhabditis elegans identified through lethal mutations. J Cell Biol. 1994;124(4):475-90.

60. Zhang C, Ruvkun G. New insights into siRNA amplification and RNAi. RNA Biol. 2012:9(8):1045-9.

61. Kagawa H, Takuwa K, Sakube Y. Mutations and expressions of the tropomyosin gene and the troponin $\mathrm{C}$ gene of Caenorhabditis elegans. Cell Struct Funct. 1997;22(1):213-8.

62. Watabe E, Ono S, Kuroyanagi H. Alternative splicing of the Caenorhabditis elegans lev-11 tropomyosin gene is regulated in a tissue-specific manner. Cytoskeleton (Hoboken). 2018;75(10):427-36.

63. Anyanful A, Sakube Y, Takuwa K, Kagawa H. The third and fourth tropomyosin isoforms of Caenorhabditis elegans are expressed in the phar$y n x$ and intestines and are essential for development and morphology. J Mol Biol. 2001;313(3):525-37.

64. Singh NN, Seo J, Ottesen EW, Shishimorova M, Bhattacharya D, Singh RN. TIA1 prevents skipping of a critical exon associated with spinal muscular atrophy. Mol Cell Biol. 2011;31(5):935-54.
65. Suzuki H, Jin Y, Otani H, Yasuda K, Inoue K. Regulation of alternative splicing of alpha-actinin transcript by Bruno-like proteins. Genes Cells. 2002;7(2):133-41.

66. Santoro M, Modoni A, Masciullo M, Gidaro T, Broccolini A, Ricci E, et al. Analysis of MTMR1 expression and correlation with muscle pathological features in juvenile/adult onset myotonic dystrophy type 1 (DM1) and in myotonic dystrophy type 2 (DM2). Exp Mol Pathol. 2010;89(2):158-68.

67. Mullen GP, Rogalski TM, Bush JA, Gorji PR, Moerman DG. Complex patterns of alternative splicing mediate the spatial and temporal distribution of perlecan/UNC-52 in Caenorhabditis elegans. Mol Biol Cell. 1999;10(10):3205-21.

68. Merz DC, Alves G, Kawano T, Zheng H, Culotti JG. UNC-52/perlecan affects gonadal leader cell migrations in C. elegans hermaphrodites through alterations in growth factor signaling. Dev Biol. 2003;256(1):173-86.

69. Wang X, Liu J, Zhu Z, Ou G. The heparan sulfate-modifying enzyme glucuronyl C5-epimerase HSE-5 controls Caenorhabditis elegans Q neuroblast polarization during migration. Dev Biol. 2015.

70. Sundararajan L, Norris ML, Lundquist EA: SDN-1/Syndecan Acts in Parallel to the Transmembrane Molecule MIG-13 to Promote Anterior Neuroblast Migration. G3 2015.

71. Sureau A, Sauliere J, Expert-Bezancon A, Marie J. CELF and PTB proteins modulate the inclusion of the beta-tropomyosin exon 6B during myogenic differentiation. Exp Cell Res. 2011;317(1):94-106.

72. Lang AE, Lundquist EA: The Collagens DPY-17 and SQT-3 Direct Anterior-Posterior Migration of the Q Neuroblasts in C. elegans. J Dev Biol 2021, 9(1).

73. Koorman T, Klompstra D, van der Voet M, Lemmens I, Ramalho JJ, Nieuwenhuize $S$, et al. A combined binary interaction and phenotypic map of C. elegans cell polarity proteins. Nat Cell Biol. 2016;18(3):337-46.

74. Banziger C, Soldini D, Schutt C, Zipperlen P, Hausmann G, Basler K. Wntless, a conserved membrane protein dedicated to the secretion of Wnt proteins from signaling cells. Cell. 2006;125(3):509-22.

75. Pan CL, Baum PD, Gu M, Jorgensen EM, Clark SG, Garriga G. C. elegans AP-2 and retromer control Wnt signaling by regulating mig-14/Wntless. Dev Cell. 2008;14(1):132-9.

76. Sawa H, Korswagen HC: Wnt signaling in C. elegans. WormBook 2013:1-30.

77. Maloof JN, Whangbo J, Harris JM, Jongeward GD, Kenyon C. A Wnt signaling pathway controls hox gene expression and neuroblast migration in C. elegans. Development. 1999;126(1):37-49.

78. Ch'ng Q, Williams L, Lie YS, Sym M, Whangbo J, Kenyon C. Identification of genes that regulate a left-right asymmetric neuronal migration in Caenorhabditis elegans. Genetics. 2003;164(4):1355-67.

79. Fire A, Xu S, Montgomery MK, Kostas SA, Driver SE, Mello CC. Potent and specific genetic interference by double-stranded RNA in Caenorhabditis elegans. Nature. 1998;391(6669):806-11.

80. Chen S, Zhou Y, Chen Y. Gu J: fastp: an ultra-fast all-in-one FASTQ preprocessor. Bioinformatics. 2018;34(17):i884-90.

81. Kim D, Paggi JM, Park C, Bennett C, Salzberg SL. Graph-based genome alignment and genotyping with HISAT2 and HISAT-genotype. Nat Biotechnol. 2019:37(8):907-15.

82. Li H, Handsaker B, Wysoker A, Fennell T, Ruan J, Homer N, et al. Genome project data processing S: the sequence alignment/map format and SAMtools. Bioinformatics. 2009;25(16):2078-9.

83. Pertea M, Kim D, Pertea GM, Leek JT, Salzberg SL: Transcript-level expression analysis of RNA-seq experiments with HISAT, StringTie and Ballgown. Nat Protoc 2016, $11(9): 1650-1667$.

84. Pertea M, Pertea GM, Antonescu CM, Chang TC, Mendell JT, Salzberg SL. StringTie enables improved reconstruction of a transcriptome from RNA-seq reads. Nat Biotechnol. 2015;33(3):290-5.

85. Liao Y, Smyth GK. Shi W: featureCounts: an efficient general purpose program for assigning sequence reads to genomic features. Bioinformatics. 2014;30(7):923-30.

86. Love MI, Huber W, Anders S. Moderated estimation of fold change and dispersion for RNA-seq data with DESeq2. Genome Biol. 2014:15(12):550.

87. Wickham H: ggplot2: Elegant Graphics for Data Analysis. SpringerVerlag New York 2009. 
88. Robinson JT, Thorvaldsdottir H, Winckler W, Guttman M, Lander ES, Getz G, et al. Integrative genomics viewer. Nat Biotechnol. 2011;29(1):24-6.

89. Thorvaldsdottir H, Robinson JT, Mesirov JP. Integrative genomics viewer (IGV): high-performance genomics data visualization and exploration. Brief Bioinform. 2013;14(2):178-92.

90. Kamath RS, Ahringer J. Genome-wide RNAi screening in Caenorhabditis elegans. Methods. 2003;30(4):313-21.

91. Kamath RS, Fraser AG, Dong Y, Poulin G, Durbin R, Gotta M, et al. Systematic functional analysis of the Caenorhabditis elegans genome using RNAi. Nature. 2003;421(6920):231-7.

92. Tamayo JV, Gujar M, Macdonald SJ, Lundquist EA. Functional transcriptomic analysis of the role of MAB-5/Hox in Q neuroblast migration in Caenorhabditis elegans. BMC Genomics. 2013;14:304.

\section{Publisher's Note}

Springer Nature remains neutral with regard to jurisdictional claims in published maps and institutional affiliations.

- fast, convenient online submission

- thorough peer review by experienced researchers in your field

- rapid publication on acceptance

- support for research data, including large and complex data types

- gold Open Access which fosters wider collaboration and increased citations

- maximum visibility for your research: over $100 \mathrm{M}$ website views per year

At BMC, research is always in progress.

Learn more biomedcentral.com/submissions 